\title{
Quantum molecular dynamics approach to heavy ion collisions: Description of the model, comparison with fragmentation data, and the mechanism of fragment formation
}

\author{
J. Aichelin, ${ }^{(a)}$ G. Peilert, ${ }^{(b)}$ A. Bohnet, ${ }^{(a)}$ A. Rosenhauer, ${ }^{(c)}$ H. Stöcker, ${ }^{(b)}$ and W. Greiner ${ }^{(b)}$ \\ ${ }^{(a)}$ Institut für Theoretische Physik der Universität Heidelberg, Heidelberg, Federal Republic of Germany \\ and Max-Planck-Institut für Kernphysik, D-6900 Heidelberg, Federal Republic of Germany \\ ${ }^{(b)}$ Institut für Theoretische Physik, J. W. Goethe-Universität, D-6000 Frankfurt am Main, Federal Republic of Germany \\ ${ }^{(c)}$ Gesellschaft für Schwerionenforschung, D-6100 Darmstadt, Federal Republic of Germany
}

(Received 21 December 1987)

\begin{abstract}
We present a detailed microscopic quantum molecular dynamic analysis of fragment formation in the reaction $\mathrm{Ne}(1.05 \mathrm{GeV} /$ nucleon $)+\mathrm{Au}$. The theoretical predictions of the total mass yield, the multiplicity distribution of clusters, their average momentum, and their angular distribution agree well with the available data. We find a rather localized hot participant zone, which predominantly emits protons and neutrons. The multiplicity of light clusters depends strongly on the impact parameter whereas the heavier fragments $A \geq 40$ result from the decay of spectator residues. Their yield can provide a good measure for the impact parameter. The hypothesis of a compound system of $A_{P}$ and $A_{T}$ nucleons which is globally heated and equilibrated is not supported by our results. Light and massive fragments occupy different regions in phase space. Semiperipheral reactions do not lead to a stopping of the projectile. We observe a power law behavior of the inclusive mass yield distribution. Its form, however, is caused by averaging over different impact parameters. This rules out inclusive mass yield distributions as candidates for revealing a possible liquid gas phase transition. Light and intermediate mass fragments are formed during the early compressional stage of the reaction. We find that the projectile causes a high density wave to travel through the target. It causes the target to fragment and transfers transverse momentum to the intermediate mass fragments. Lighter fragments receive additional momentum transfer due to $n-n$ collisions.
\end{abstract}

\section{INTRODUCTION}

The formation of medium-mass clusters $5 \leq A \leq 30$ in medium- and high-energy heavy ion collisions currently is a topic of great experimental and theoretical interest. Up to now very little is known about the underlying mechanism of fragment formation-only that it has a high threshold ( $E_{\text {beam }}>25 \mathrm{MeV} /$ nucleon) and therefore became accessible for experiments only recently. Proton induced reactions ${ }^{1}$ exhibit a power law behavior $\left[\sigma(A)=A^{-\tau}\right]$ of the mass yield curve, a functional form which is expected for a system close to the transition between the liquid and vapor phases. ${ }^{1,2}$ The same form of the mass yield curve has later also been found in heavy ion induced reactions. ${ }^{3}$ This similarity led to the suggestion that the observed shape of the inclusive mass yield curve presents evidence for the occurrence of this phase transition in nuclear collisions. ${ }^{3,4}$ This conjecture started a vivid debate which is still not settled. Theoretical investigations, however, have demonstrated that the mass yield curve is rather insensitive to different reaction mechanisms proposed: statistical or thermodynamical models without Coulomb interaction, ${ }^{4-6}$ or including it, ${ }^{7,8}$ describe the observed mass yield equally well as models in which the system does not come to a global ${ }^{9}$ or at least to a local equilibrium prior to fragmentation. The latter class of phenomenological models contains approaches in which the fragmentation is assumed to be similar to the percolation of a finite lattice ${ }^{10,11}$ or like the shattering of glass. ${ }^{12-14}$
Another type of model treated the nucleus-nucleus collision as a two step process: an early compression phase leading to a global thermodynamical equilibrium of the whole system which then can be completely described by two variables; a temperature $T$ and a density $\rho$. It serves as the initial condition for the subsequent expansion phase which is treated microscopically, either by using the time-dependent Hartree Fock (TDHF) (Ref. 15) equations or by applying classical molecular dynamics. ${ }^{16}$ The essential result of these approaches was the observation that fluctuations of the phase space density at the beginning of the expansion reflect in the distribution of the observed clusters.

Cluster formation has first been treated in a model which describes the complete time evolution of the reaction by applying a potential model to the final phase space distribution of a Boltzmann-Uehling-Uhlenbeck ${ }^{17}$ (BUU) calculation or by applying a phase space coalescence model to the Vlasov-Uehling-Uhlenbeck ${ }^{18}$ (VUU) model. Both approaches aimed at a removal of their contribution to the primordial proton spectra. In this single particle theory, which has also been dubbed LandauVlasov approach, ${ }^{19}$ one starts out with well separated projectile and target nuclei which are boosted towards each other. The projectile and target contain $A_{T}$ and $A_{P}$ pointlike particles, representing the nucleons, which move on curved trajectories under the influence of a selfconsistent mean field. In addition, they perform collisions among each other which respect the Pauli principle. These calculations describe very successfully single 
particle observables, ${ }^{17,18}$ the creation of $\pi$ 's, ${ }^{18}$ deuterons, ${ }^{20}$ kaons, ${ }^{21}$ and global $(4 \pi)$ observables like the flow angle or the collective momentum transfer. ${ }^{18}$

In actual calculations many simulations (typically 100) have to be performed simultaneously to damp the large fluctuations of the mean field in a single simulation by ensemble averaging. Unavoidably the ensemble averaging smears out fluctuations and correlations of an individual simulation, which are assumed to be essential for the formation of the many clusters observed in a nucleusnucleus collision. Therefore, this type of approach cannot be utilized to study the formation of medium mass fragments.

Nonetheless, several modifications of the BUU approach were recently advanced to incorporate fragment formation. None of them, however, overcame the above-mentioned problem. Bauer et al. ${ }^{22}$ reduced the nucleon-nucleon cross section by a factor of 100 . For compensation, when a collision occurs, $2 \times 99$ nucleons in the vicinity of the actual collision partners are scattered as well. Between collisions particles move in the ensemble averaged mean field. This method has three crucial problems: first of all the result depends on how to select the $2 \times 99$ nucleons out of total number of nucleons ( $100 A_{\text {target }}+100 A_{\text {projectile }}$ ) present, and how to determine the partners in the individual nucleon-nucleon collision. Secondly, the propagation in the mean field washes out part of the fluctuation caused by the collisions, and thirdly the Pauli blocking is not well defined because the whole neighborhood of the collision partners in phase space is changed at the same time.

Das Gupta et $a .^{23}$ divide the reaction in two steps. An initial step, where collisions are dominant, and an expansion, where the mean field provides the clustering of the nucleons. The initial stage is described by a highenergy cascade calculation. It generates the input for the subsequent mean field calculation which describes the expansion. If the clusters were made only from nucleons whose time evolution is initially dominated by collisions (i.e., "fireball nucleons"), this method would be reasonable. If the clusters are predominantly spectator nucleons, this method has to fail, because for the time evolution of spectator nucleons the field is also dominant in the first stage. As we will see, we find that clusters predominantly contain spectator nucleons.

Recently we developed a microscopic model [dubbed quantum molecular dynamics ${ }^{24}$ (QMD)] which allows to investigate the formation of clusters during a heavy ion collision in a consistent $N$-body treatment. In this approach the nucleons are represented by Gaussian wave packets which interact by mutual two and three body forces. This model simulates heavy-ion reactions on an event-by-event basis and, as a consequence, preserves correlations and fluctuations. Therefore, it allows to address the formation of clusters. This theory approximates the complete quantal theory in the sense that the nucleons are spread out in coordinate and momentum space with a Gaussian distribution. The width of the distribution, however, is kept constant. Collisions, which respect the Pauli principle, are incorporated in a similar way as in BUU or VUU. This model furnishes the com- plete $N$-body information of the event, i.e., it allows to calculate the triple differential cross section $d^{3} \sigma /$ $d E d \Omega d Z$ as well as correlations among the particles like the collective flow of fragments. Within this model Peilert et al. ${ }^{25}$ have investigated the formation and flow of complex fragments in heavy symmetric systems $(\mathrm{Au}+\mathrm{Au}$ and $\mathrm{Nb}+\mathrm{Nb})$. Rosenhauer et al. have studied the influence of medium effects and the nuclear equation of state on nuclear stopping and flow. ${ }^{26} \mathrm{We}$ want to mention that Beauvais et al. ${ }^{27}$ developed a similar approach which is, however, based on a density dependent mean field.

The description of the model is given in Sec. II. Here we also report on the numerical tests performed to show that the model is appropriate to address the formation of clusters.

The crucial test for such a model is the comparison with experiments. There is a wealth of data of inclusive mass yields which, as already mentioned, are unfortunately not very sensitive to the reaction mechanism. Data with more detailed information, however, are rather scarce. We are only aware of two experiments: Warwick et al. ${ }^{28}$ measured the triple differential cross section $d^{3} \sigma / d E d \Omega d Z$ of medium mass fragments, and Waddington and Freier ${ }^{29}$ have recorded the exclusive mass yield for the reaction $\mathrm{Au}+$ emulsion at $1 \mathrm{GeV} /$ nucleon. The primary goal of this experiment was the search for anomalons. The results as far as of interest for the multifragmentation of the $\mathrm{Au}$ nucleus were recently analyzed. ${ }^{30}$

In the present paper we study the reaction 1050 $\mathrm{MeV} /$ nucleon $\mathrm{Ne}+\mathrm{Au}$, and compare the theoretical results and experimental ${ }^{28}$ data in Sec. III. Since some nuclei of the emulsion $(\mathrm{C}, \mathrm{O})$ are similar to $\mathrm{Ne}$, we also compare with the experimental data of Waddington and Freier. ${ }^{29}$ This is followed by a study of the impact parameter dependence of the quantities of interest and the time evolution of the clusters. We then address the question whether a (global or local) equilibrium is achieved in the course of the reaction by looking at the mean velocity and the temperature of different classes of clusters and by studying correlation between entrance and exit channel. Finally, we investigate what causes the nucleus to fragment into many pieces. The conclusions are given in Sec. IV.

\section{THE MODEL}

The structure of the QMD approach can be best discussed when we start out with the classical molecular dynamics approach, ${ }^{31-33}$ i.e., with the assumption that nucleons behave like classical particles which interact by long range forces. In this case the scattering angle is uniquely determined by the impact parameter and there is no Pauli blocking. The classical molecular dynamics is a $N$-body theory. All information about the system is contained in the solution of the $N$ body Liouville equation. If the potentials are conservative, the solution is time reversible (i.e., replacing at $t=\tau$ all momenta $p$ by $-p$ the system is again in its initial configuration at $t=2 \tau)$. No information is lost in the course of the time 
evolution.

This is in sharp contrast to the solution of the Boltzmann equation - a one body theory, which can be derived from the Liouville equation by integrating over the phase space coordinates of $N-1$ particles and closing the equation at the level of the one body phase space density. The closure is obtained by the Stosszahlansatz $\rho_{2}\left(x_{1}, x_{2}, p_{1}, p_{2}\right)=\rho_{1}\left(x_{1}, p_{1}\right) \rho_{1}\left(x_{2}, p_{2}\right)$. If one wants to include a self-consistent mean field one has to choose a specific ansatz for the three body density matrix since beside the scattering partners a third particle is required which provides the mean field. The prize for closing the equation on the one particle level is the loss of information due to the neglect of correlations. This equation is not time reversible - the system moves toward thermal equilibrium and entropy is produced. Although the BUU or VUU approaches are genuine $N$-body theories-one follows the positions and momenta of all $N$ particles and thus calculates the time evolution of the $N$-body density matrix - the numerical procedure to solve these equations, i.e., the averaging over many ensembles, mixes correlations and fluctuations among different ensembles and renders them useless. The predictive power of the BUU or VUU approach is therefore limited to one body observables. As we will see the ensemble averaging is not required in the case of the QMD approach, and hence, the fluctuations and correlations are preserved.

Important quantum features are included in the QMD approach: collisions among nucleons are Pauli blocked when the scattered nucleons would enter already occupied or partially occupied phase space regions. Furthermore, the scattering amplitude does not relate the scattering angle with the impact parameter in a unique way: The square of the scattering amplitude is identified as a probability distribution. The scattering angle as well as the blocking of collisions which brings nucleons in a partially occupied phase space region are treated statistically. This procedure destroys the time reversibility of the classical equation. However, the model is still the solution of the $N$-body equation, not a reduction to the one body level, and-as a consequence-also describes the time evolution of all correlations. The QMD model can therefore be viewed as a classical $N$-body approach in which quantum corrections up to the second order in the rank of the density matrix are incorporated. ${ }^{34}$

These microscopic models are chaotic in the sense that the two neighboring phase space points in the $A_{T}+A_{P}$ dimensional phase space diverge exponentially as a function of time. In a quantum system we cannot determine the impact parameter more precisely than $\Delta b>\hbar / \Delta P$. Instead of varying the impact parameter over this region we initialize the nuclei differently by drawing different random numbers for the position and the momentum of the particles. This will be explained in the next section.

\section{A. Initialization}

To simulate heavy ion collisions one faces two critical points: the initialization of the projectile and target nuclei and the time evolution of the $A_{T}+A_{P}$ system. Let us first concentrate on the first topic.

When comparing quantal (TDHF) and the classical (Vlasov) mean field systems ${ }^{35}$ we found an almost identical time evolution of the nuclear density for beam energies larger than $25 \mathrm{MeV} /$ nucleon. Although the differential equations for the time evolution of the classical and quantal system are almost identical this is quite surprising because of the different initial states. The initial density of the former calculation is given by a Slater determinant whereas the Vlasov equation starts with pointlike particles randomly distributed in a sphere of the radius $r=1.14 A^{1 / 3}$, corresponding to a normal nuclear matter density of 0.16 nucleons $/ \mathrm{fm}^{3}$. From these results we have concluded that, at the energies considered, the detailed form of the wave function has only a minor influence on the time evolution of the bulk properties of the system, provided it fulfills minimal requirements, like providing a roughly constant density over the proper region in coordinate space. Therefore, it is reasonable to start with a form of the wave function which is easy to handle. An obvious candidate is a Gaussian form of the wave function of a nucleon $i$ :

$$
\psi_{i}(\mathbf{r}, \mathbf{p}, t)=\frac{1}{(2 \pi L)^{3 / 4}} e^{-\left[\mathbf{r}-\mathbf{r}_{i 0}(t)\right]^{2} / 4 L} e^{i \mathrm{pr}} .
$$

To keep the formulation as close as possible to the classical transport theory, we use Wigner densities instead of working with wave functions. For details we refer to Ref. 36. The Wigner representation of our $A_{T}+A_{P}$ particle system is given by

$$
\begin{aligned}
f(\mathbf{R}, \mathbf{p}, t) & =\sum_{i} \frac{1}{(2 \pi \hbar)^{3}} \int e^{-i \mathbf{p r}_{12} / \hbar} \psi_{i}\left(\frac{\mathbf{R}+\mathbf{r}_{12}}{2}, t\right) \psi_{i}^{*}\left(\frac{\mathbf{R}-\mathbf{r}_{12}}{2}, t\right) d^{2} \mathbf{r}_{12} \\
& =\sum_{i} \frac{1}{(\pi \hbar)^{3}} \exp \left\{-\left[\mathbf{R}-\mathbf{R}_{\mathbf{i}}(t)\right]^{2} / 2 L-\left[\mathbf{p}-\mathbf{p}_{\mathbf{i}}(t)\right]^{2} 2 L / \hbar^{2}\right\} \\
& =\sum_{i} f_{i}(\mathbf{R}, \mathbf{p}, t)
\end{aligned}
$$

where $L=1.08 \mathrm{fm}^{2}$ corresponding to a root mean square radius of the nucleons of $1.8 \mathrm{fm}$. Wigner representations behave very much like classical phase space densities, but they can be negative. The Wigner representation of our Gaussian wave packets obeys the uncertainty relation $\Delta x \Delta p_{x}=\hbar / 2$. The densities in coordinate and in 
momentum space are given by

$$
\begin{aligned}
n(\mathbf{R}, t) & =\int f(\mathbf{R}, \mathbf{p}, t) d^{3} p \\
& =\sum_{i} \frac{1}{(2 \pi L)^{3 / 2}} e^{-\left[\mathbf{R}-\mathbf{r}_{i 0}(t)\right]^{2} / 2 L},
\end{aligned}
$$

and

$$
g(\mathbf{p}, t)=\int f(\mathbf{R}, \mathbf{p}, t) d^{3} R
$$

A random choice of the centers of the $A_{T}+A_{P}$ Gaussians in coordinate and momentum space is not sufficient to maintain the stability of the nuclei for a sufficiently long time. Due to fluctuations, a limited sequence of random numbers does not create the ground state of a nucleus but rather a metastable excited state which decays by emission of nucleons. The time span for which the nucleus is stable implies an upper limit to the excitation energy which can be tolerated.

Eigenstates of a Hamiltonian have to fulfill the uncertainty relation. The variance $\Delta x \Delta p_{x}$ of two neighboring eigenfunctions is separated by $\hbar / 2$, i.e., each level fills a volume of $h^{3}$ in phase space. If a system is in its ground state, the phase space is densely filled up to a maximum value in coordinate and momentum space. Loosely spoken, there is no hole in the phase space. This property of the ground state we employ to initialize the nuclei. First we determine the position the nucleons in a sphere of the radius $R=1.14 A^{1 / 3}$. We draw random numbers but reject those which would position the centers of two nucleons closer than $r_{\min }=1.5 \mathrm{fm}$. The next step is to determine the local density at the centers of all nucleons generated by all the other nucleons. Applying a local Fermi gas approximation, we calculate the local Fermi momentum. Finally the momenta of all particles are chosen randomly between zero and the local Fermi momentum. We then reject all random numbers which position two particles closer in phase space than $\left(r_{i}-r_{j}\right)^{2}\left(p_{i}-p_{j}\right)^{2}=d_{\min }$. Typically only 1 out of 50000 initializations is accepted under the present criteria. The accepted configurations are quite stable: usually no nucleon escapes from the nucleus in $300 \mathrm{fm} / c$, as we will see later. This procedure also ensures that the nuclei have the proper root mean square radii in coordinate and momentum space. The computer time required for the initialization is short compared to the time needed for the propagation.

\section{B. Propagation}

Nuclei which have been successfully initialized are then boosted towards each other with the proper center of mass velocity using relativistic kinematics. During the calculation we keep the width of the wave function fixed. This drawback represents the limit of the theory. For the results presented it is not a major concern: we have checked the influence of the width on the mass yield by doubling it and found differences on the $20 \%$ level. The mean values $\left(r_{i 0}, p_{i 0}\right)$ are propagated under the influence of mutual two and three body interactions as described by the Poisson brackets

$$
\dot{p}_{i 0}=\left\{r_{i 0}, H\right\}=\left\{r_{i 0}, T+U\right\},
$$

and

$$
\dot{r}_{i 0}=\left\{p_{i 0}, H\right\}=\left\{p_{i 0}, T+U\right\} .
$$

Here $T$ is the total kinetic energy and $U$ is the total potential energy of all nucleons. These differential equations are solved using an Eulerian integration routine with a fixed time step $\Delta t$,

$$
\begin{aligned}
p_{i 0}(n+1)= & p_{i 0}(n)-\nabla_{r} U_{i}\left(n+\frac{1}{2}\right) \Delta t, \\
r_{i 0}\left(n+\frac{1}{2}\right)= & r_{i 0}\left(n-\frac{1}{2}\right)+\frac{p_{i 0}(n)}{\left[p_{i 0}(n)^{2}+m_{i}^{2}\right]^{1 / 2}} \Delta t \\
& +\nabla_{p} U_{i}(n) \Delta t .
\end{aligned}
$$

The particles interact via two and three body interactions, not via a mean field as in the BUU/VUU calculations. This is essential if the fluctuations and correlations are to be preserved. We assume that the short range interactions between the nucleons accounts for the bulk properties. This Skyrme-type interaction is supplemented by a long range Yukawa interaction which is necessary to reproduce surface effects and an effective charge Coulomb interaction, where all particles have a charge $Z / A$.

Our total interaction is given by

$$
H=V^{\text {loc }}+V^{\mathrm{Yuk}}+V^{\mathrm{Coul}},
$$

where the different terms are given by

$$
\begin{aligned}
& V^{\mathrm{loc}}=t_{1} \delta\left(r_{1}-r_{2}\right)+t_{2} \delta\left(r_{1}-r_{2}\right) \delta\left(r_{1}-r_{3}\right), \\
& V^{\mathrm{Yuk}}=t_{3} \frac{e^{-\left|r_{1}-r_{2}\right| / m}}{\left|r_{1}-r_{2}\right| / m},
\end{aligned}
$$

with $m=0.8 \mathrm{fm}$. The potential acting on particle 1 is hence given by the expectation value of the two and three body interaction

$$
U_{1}(t)=U_{1}^{(2)}(t)+U_{1}^{(3)}(t)
$$

where the upper index refers to the two and three body interaction, respectively. The two body interaction is given by

$$
\begin{aligned}
& U_{1}^{(2)}(t)=\sum_{i, i \neq 1} \int f_{1}\left(R_{1}, p_{1}, t\right) f_{i}\left(R_{2}, p_{2}, t\right) \\
& \times V^{(2)}(1,2) d^{3} R_{1} d^{3} p_{1} d^{3} R_{2} d^{3} p_{2} \\
&= U_{1 \text { loc }}^{(2)}+U_{1 \text { Yuk }}^{(2)}+U_{1 \text { Coul }}^{(2)},
\end{aligned}
$$

with

$$
U_{1 \text { loc }}^{(2)}=\frac{t_{1}}{(4 \pi L)^{3 / 2}} \sum_{i \neq 1} e^{\left(r_{10}-r_{i 0}\right)^{2} / 4 L},
$$

and 


$$
U_{1 \mathrm{Yuk}}^{(2)}=t_{3} \sum_{i \neq 1} \frac{e^{L / m^{2}}}{r_{1 i} / 2 m}\left\{e^{-r_{1 i} / m}\left[1-\Phi\left(\frac{\sqrt{L}}{m}-\frac{r_{1 i}}{2 \sqrt{L}}\right)\right]-e^{r_{1 i} / m}\left[1-\Phi\left(\frac{\sqrt{L}}{m}+\frac{r_{1 i}}{2 \sqrt{L}}\right)\right]\right\},
$$

where $\Phi(x)$ is the error function. The three body potential is given by

$$
\begin{aligned}
U_{1}^{(3)} & =t_{2} \sum_{i, j ; j, i \neq 1, i \neq j} \int f_{1}\left(R_{1}, p_{1}, t\right) f_{i}\left(R_{2}, p_{2}, t\right) f_{j}\left(R_{3}, p_{3}, t\right) V^{(3)} d^{3} R_{1} d^{3} p_{1} d^{3} R_{2} d^{3} p_{2} d^{3} R_{3} d^{3} p_{3} \\
& =\frac{t_{2}}{(2 \pi L)^{3} 3^{3 / 2}} \sum_{i, j ; j, i \neq 1, i \neq j} \exp \left[\left(r_{10}-r_{i 0}\right)^{2}+\left(r_{10}-r_{j 0}\right)^{2}+\left(r_{j 0}-r_{i 0}\right)^{2}\right] / 6 L \\
& \approx \frac{t_{2}}{(2 \pi L)^{3} 3^{3 / 2}} \sum_{i, j ; j, i \neq 1} \exp \left[\left(r_{10}-r_{i 0}\right)^{2}+\left(r_{10}-r_{j 0}\right)^{2}\right] / 4 L \\
& =\frac{t_{2}}{(2 \pi L)^{(v-1)^{3 / 2}} v^{3 / 2}} h^{v-1}
\end{aligned}
$$

with $v=3$ and

$$
h=\sum_{i, i \neq 1} e^{\left[\left(r_{10}-r_{i 0}\right)^{2}\right] / 4 L} .
$$

The expectation value of the total energy is

$$
E=\sum_{i} T_{i}+\frac{1}{2} \sum_{i, j, i \neq j} U_{i j}^{(2)}+\frac{1}{3 !} \sum_{i, j, k, i \neq j, i, j \neq k} U_{i j k}^{(3)}
$$

Next we have to determine the parameters $t_{1}-t_{3}$. We start out from the observation that in nuclear matter, where the density is constant, $U^{(2)}$ is directly proportional to $\rho / \rho_{0}$. In spin saturated nuclear matter the three body interaction is equivalent to a density dependent two body interaction where the density has to be taken at the center of mass of the interacting partners. The assumption of the density dependence of the nuclear potentials is probably closer to the physical reality than the assumption of a three body interaction which has been found to be small. If we assume that the density does not vary substantially over the distance of the two body interaction $U^{(3)}$ is then proportional to $\left(\rho / \rho_{0}\right)^{2}$. This observation allows to relate the desired parameters to nuclear matter properties. However, we want to stress that for the actual propagation always the explicit two and three body interactions are used and not the nuclear matter potentials. This is important since the equivalence of both is only true in nuclear matter, not in finite nuclei.

This equivalence can be used to connect the parameters $t_{1}-t_{3}$ with nuclear matter properties, i.e., the nuclear equation of state. For this reason, our approach allows to investigate in detail how a given nuclear equation of state shows up in different observables in a heavy ion reaction. In nuclear matter our two and three body interaction are up to small corrections equivalent to a density dependent interaction of the form

$$
U^{\mathrm{loc}}=\alpha\left(\frac{\rho}{\rho_{0}}\right)+\beta\left(\frac{\rho}{\rho_{0}}\right)^{2}
$$

This potential has two free parameters which can be fixed by the requirement that at normal nuclear matter density the average binding energy is $-16 \mathrm{MeV}$ and the total energy has a minimum at $\rho_{0}$. The adjustment of the two parameters fixes the compressibility as well. In order to investigate the influence of different compressibilities one can generalize the potential to

$$
U=\alpha\left(\frac{\rho}{\rho_{0}}\right)+\beta\left(\frac{\rho}{\rho_{0}}\right)^{\gamma} .
$$

Now we have in addition a third parameter which allows to fix the compressibility independently from the other quantities. This generalization can be translated back to the nucleon-nucleon potential in a unique way by identifying $v$ with $\gamma$.

The parameter $\alpha$ contains contributions from the local two body interaction as well as from the Yukawa interaction. The latter can be obtained by the Taylor expan$\operatorname{sion}^{38}$

$$
\begin{aligned}
U_{\mathrm{Yuk}} & \sim \int d^{3} r \int d^{3} r^{\prime} \frac{e^{-\left|r-r^{\prime}\right| / m}}{\left|r-r^{\prime}\right| / m} \rho(r) \rho\left(r^{\prime}\right) \\
& =4 \pi m^{3}\left\{\int d^{3} r \rho^{2}(r)+m^{2} \int d^{3} r\left[\rho(r) \nabla^{2} \rho(r)\right]\right\} .
\end{aligned}
$$

Hence $\alpha$ is given by

$$
\alpha \sim t_{1}-4 \pi m^{3} t_{3} .
$$

Note that $t_{1}^{\mathrm{QMD}}=\frac{3}{4} t_{1}^{\mathrm{TDHF}} \cdot 38$

The relative weight between $t_{1}$ and $t_{3}$ as well as the parameter $m$ are adjusted to obtain reasonable binding energies of finite nuclei. We find that $t_{2}=10 \mathrm{MeV}$ and $m=0.8 \mathrm{fm}$ give $E / A=6-14 \mathrm{MeV}$ for $A=7-200$. The coefficients of proportionality between $\alpha$ and $t_{1}$ and $\beta$ and $t_{2}$, respectively, are determined numerically.

In Fig. 1 we display the density dependence of the ground state energy per particle in nuclear matter for two 


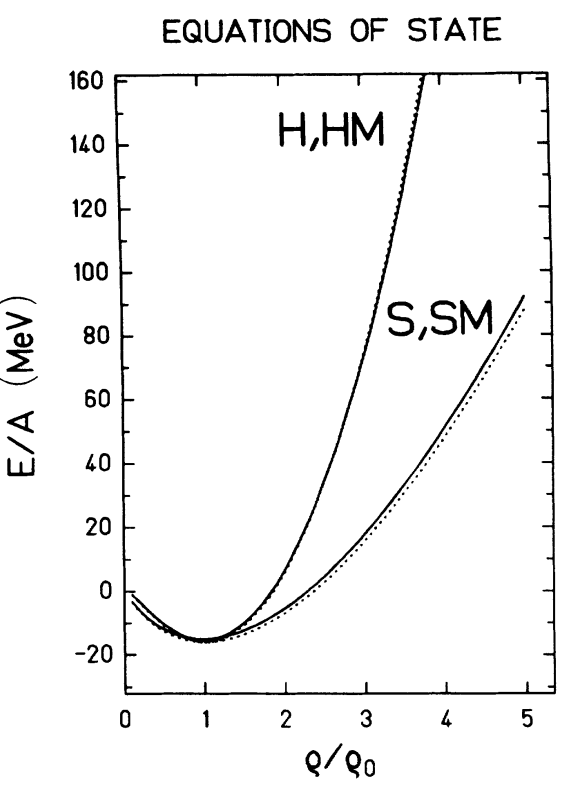

FIG. 1. Equation of state. The density dependence of the energy per particle in nuclear matter at temperature $T=0$ is displayed for our four different sets of parameters.

different sets of parameters.

Set $1(H)$ : Hard equation of state, compressibili$\mathrm{ty}=380 \mathrm{MeV}$

$$
\alpha=-124 \mathrm{MeV}, \quad \beta=70.5 \mathrm{MeV}, \quad \gamma=2 .
$$
$\mathrm{MeV}$

Set $2(S)$ : Soft equation of state, compressibility $=200$

$$
\alpha=-356 \mathrm{MeV}, \quad \beta=303 \mathrm{MeV}, \quad \gamma=\frac{7}{6} \text {. }
$$

We just want to mention that this form of the nucleon-nucleon potential can easily be supplemented by a momentum dependent interaction $(H M, S M) .{ }^{37}$ For a given compressibility this does not change the energy/nucleon in nuclear matter up to 4 times nuclear matter density, but has important consequences concerning the dynamics of a heavy ion reaction. However, as far as multifragmentation is concerned, the influence is small. $^{25}$

\section{Collisions}

The scattering of nucleons in nuclear matter in the low density expansion is described in terms of the reaction $g$ matrix $^{39}$

$$
g(w)=V+V \frac{Q}{w-e+i \delta} g(w),
$$

where the Pauli operator $Q$ projects on unoccupied states only and $e$ is the energy of the intermediate state

$$
e=p_{1}^{2} / 2 m+p_{2}^{2} / 2 m+U\left(p_{1}\right)+U\left(p_{2}\right) .
$$

At high energies the influence of the Pauli blocking is small and the kinetic energy is large as compared to the potential $U$. Then the imaginary part of the reaction matrix becomes identical to the transition matrix which de- scribes the scattering between two free nucleons. We assume to be in an energy region where we can neglect the Pauli blocking of the intermediate states and treat the Pauli blocking of the final state explicitly. This is probably a reasonable approximation for beam energies larger than $200 \mathrm{MeV} /$ nucleon. Of course it would be highly desirable to extend the in-medium corrected scattering amplitudes which are available for an equilibrated environment of the scattering partners and amount to a $30 \%$ reduction of $\sigma_{\text {free }}$ (Ref. 40) to the nonequilibrium situation as found during the early state of heavy ion reactions. For a detailed discussion of these in-medium effects we refer to Ref. 26. We neglect the in-medium effects of the intermediate states and use the measured free nucleon-nucleon scattering cross section. The effective cross section is however smaller due to the Pauli blocking. We use the parametrization of Cugnon ${ }^{41}$ for the elastic as well as for the inelastic part.

Two nucleons can collide if they come closer than $r=\sqrt{\sigma / \pi}$. The scattering angles of the single nucleonnucleon collisions are randomly chosen such that the sum of the scattering angles of all collisions agrees with the measured angular distribution for elastic collisions.

Inelastic collisions lead to the formation of deltas which can be reabsorbed by the inverse reaction. We do not incorporate free ( $s$ wave) pions here, unlike in the VUU approach. ${ }^{18}$

\section{Pauli blocking}

Whenever a collision has occurred, we check the phase space around the final states of the scattering partners. For simplicity we assume that each nucleon occupies a sphere in coordinate and momentum space. This trick yields the same Pauli blocking ratio as an exact calculation of the overlap of the Gaussians but is much less time consuming to calculate. We calculate which percentage $P_{1}$ and $P_{2}$ of the final phase space for each of the two scattering partners is already occupied by other nucleons. The collision is then blocked with a probability

$$
P_{\text {block }}=P_{1} P_{2} \text {, }
$$

and, correspondingly, is allowed with the probability $1-P_{\text {block}}$. Whenever a collision is blocked, we replace the momentum of the scattering partners by the value it had prior to the scattering. Care is taken for nucleons which are close to the surface of the many nucleon system where the above description includes also portions of phase space which are classically forbidden as a consequence of energy conservation. For a nucleus in its ground state, where all collisions should be blocked, we obtain an averaged blocking probability $\left\langle P_{\text {block }}\right\rangle$ of 0.96 . This determines the low energy limit of our theory: aiming at $25 \%$ artificial collisions, i.e., collisions which are due to an insufficient Pauli blocking, we can tolerate only beam energies at which no more than $84 \%$ of the collisions are blocked. Therefore, $E_{\mathrm{lab}}=20 \mathrm{MeV} /$ nucleon is at the moment the lower bound of our approach. Presently we are working on an extension of this model to even lower energies. 


\section{E. Numerical tests}

One basic requirement that the present model has to fulfill is the stability of the nuclei on a time scale compatible with the time span required for a nucleus-nucleus collision to occur. High-energy collisions $\left(E_{\text {lab }}>500\right.$ $\mathrm{MeV} /$ nucleon) require less than $100 \mathrm{fm} / c$ as far as single particle properties are concerned. However, it turns out that in order to investigate the fragmentation process in heavy ion collisions we have to follow the reaction for a considerable longer time. This is so because instable fragments are formed which have sufficient excitation energy to decay on a longer time scale.

Figure 2 shows the time evolution of the root mean square radii of two nuclei, $\mathrm{Ne}$ and $\mathrm{Au}$, for four different time steps $\Delta t$. We see oscillations around the mean value but no nucleons are emitted. These oscillations are not surprising because the nuclei are not in their quantal ground state rather they have a small excitation energy.

To visualize the time evolution of one nucleon in the field generated by all the others, we display in Fig. 3 the trajectory of a single nucleon in a gold nucleus for a time span of $200 \mathrm{fm} / c$. To visualize the size of the system we show also a sphere of radius $r=1.3 \times 197^{1 / 3}$. As one can see the mutual interactions confine the constituents well to this radius, hence the nuclear shape can be generated by interacting nucleons with Fermi motion in the present approach.

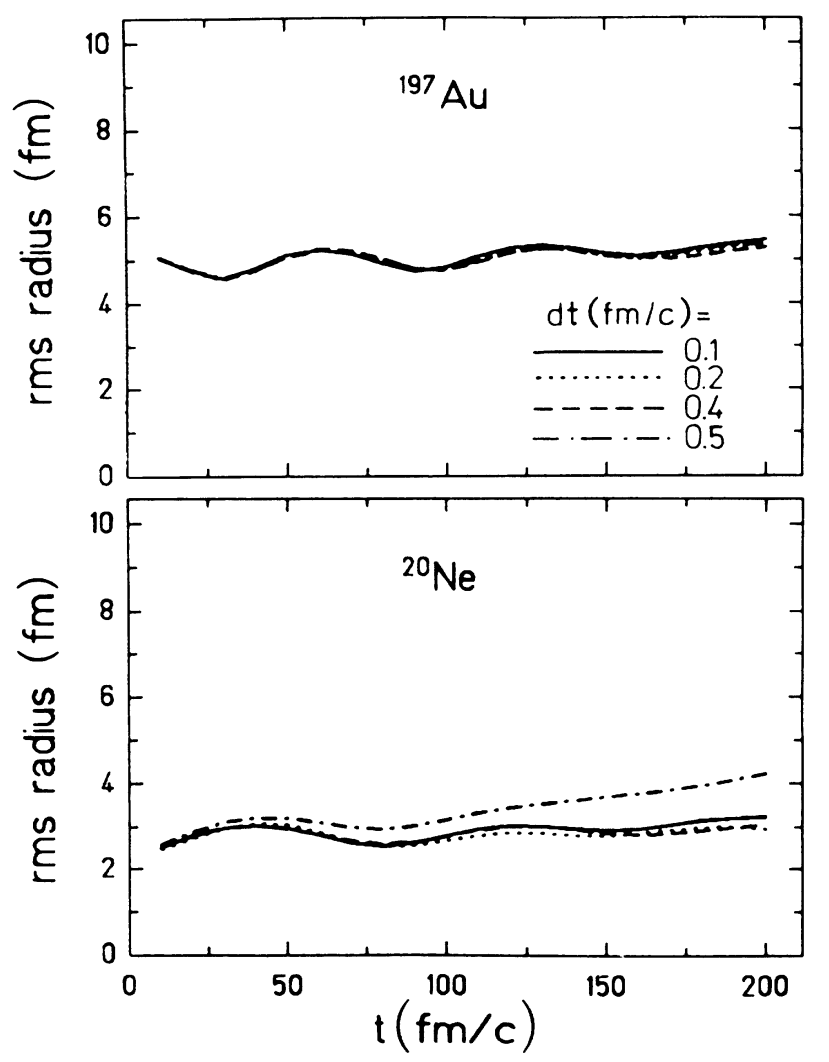

FIG. 2. Stability of clusters. The time evolution of the root mean square radii of two nuclei, $\mathrm{Ne}$ and $\mathrm{Au}$, for four different time step sizes $\Delta t$.

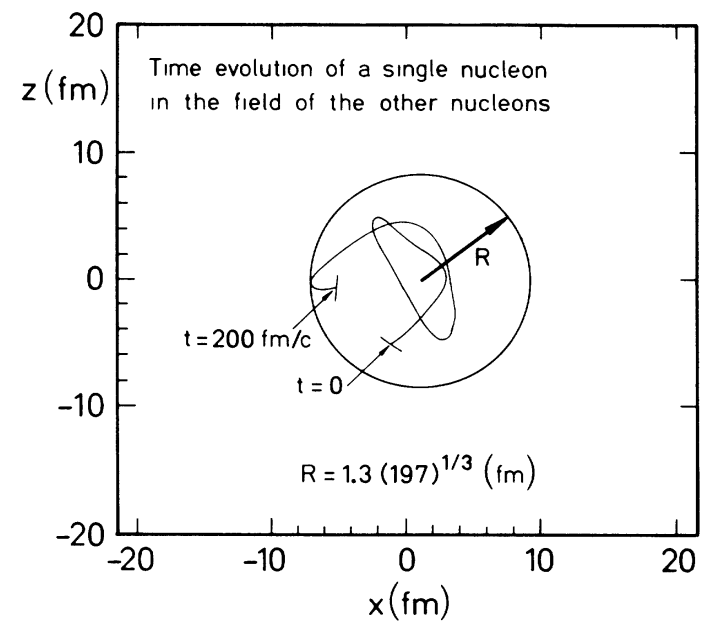

FIG. 3. The trajectory of a single nucleon in the field of 196 others is displayed for a time span of $200 \mathrm{fm} / c$. To visualize the size of the system we show also a sphere of radius $r=1.14 \times 197^{1 / 3}$.

\section{MULTIFRAGMENTATION}

Although the fragmentation of heavy target nuclei is a well established phenomenon, there are only two experiments known to us which have measured more than just the inclusive mass yield of the fragmentation products. We compare in Sec. III A the results of the present QMD approach with detailed data on fragment production from the reaction $\mathrm{Ne}(1050 \mathrm{MeV} /$ nucleon $)+\mathrm{Au}^{28}$ In this experiment triple differential cross sections $d^{3} \sigma / d E d \Omega d Z$ were recorded and in addition the associated multiplicity of fast particles has been measured. These fast particles provide a tag for the violence (impact parameter) of the reaction. To calculate the triple differential cross section $d^{3} \sigma / d E d \Omega d Z$ is beyond the feasibility of present-day computers. We therefore have to restrict ourselves to mean values and to the angular distribution

$$
d \sigma / d \Omega=\sum_{Z} \int d E d^{3} \sigma / d E d \Omega d Z
$$

In addition to the associated charged particle multiplicity the correlations between jets of light fragments and the target rapidity fragment were measured. In this way the strong azimuthal (anti-) correlation which has been predicted by hydrodynamics ${ }^{42}$ ("bounce off") has been observed. ${ }^{28}$

However, no correlation between complex fragments have been measured in this experiment. The multiplicity of complex fragments was determined in an emulsion experiment corresponding to approximately the same system. $^{29}$ To study the fragment multiplicities we supplement our analysis by calculating the distribution of the number of heavy fragments obtained in a single collision. We then compare this distribution with the data for $\mathrm{Au}+$ emulsion at $1 \mathrm{GeV} /$ nucleon, where all charged remnants of the gold nucleus were recorded. ${ }^{29}$ Special acceptance windows are applied. They exclude peri- 
pheral reactions and collisions with the heavy constituents of the emulsion. To our knowledge this is all the available information on fragmentation in heavy ion induced reactions beyond the inclusive mass yield.

Of course the theory provides in principle much more information than the experiment because it also yields information about the time evolution of the system in the coordinate and momentum space. In Sec. III B we demonstrate what one can learn from this additional information. In particular we investigate the impact parameter dependence of the mass yield and the momentum space distribution. Furthermore, we search for correlations between initial and final state to see whether the system comes to a global equilibrium state in the course of the reaction (where all remembrance on the initial distribution would be lost). This is of special importance for the validity of those models which assume global thermal equilibrium. ${ }^{7,8,15,16}$

Although in every central collision the target nucleus fragments into several large pieces, the actual fragmentation pattern of a single nucleus nucleus collision is determined by fluctuations. We investigate in Sec. III C in detail what causes pieces of nuclear matter to break off and to form a fragment.

\section{A. Comparison with experimental data}

We studied the reaction $\mathrm{Ne}(1050 \mathrm{MeV} /$ nucleon $)+\mathrm{Au}$ at four different impact parameters $(b=1,3,5$, and $7 \mathrm{fm})$. Even larger impact parameters do not produce a significant amount of fragments in the most interesting range $5 \leq A \leq 30$. For the two most central impact parameters we have calculated 360 events whereas for the larger impact parameters we restricted ourselves to 180 simulations. We found about a $10 \%$ change of the cluster yield for the different nuclear equations of state. One simulation requires 1 min CPU time on a Cray-1 computer.

The reactions were followed for $300 \mathrm{fm} / c$ $\left(1 \times 10^{-21} \mathrm{sec}\right)$. This is a very long time as compared to the time the neon projectile needs to cross the target $(20$ $\mathrm{fm} / \mathrm{c}$ ) and even longer than was required for simulating reactions at $25 \mathrm{MeV} /$ nucleon. ${ }^{17}$ However, as we will see, the mass distribution continuously changes up to this time as a consequence of the long decay time of moderately excited heavy clusters. Nucleons are considered to be part of a cluster if finally (at $t=300 \mathrm{fm} / c$ ) at least one other nucleon is closer than $r_{\min }=3 \mathrm{fm}$. No cuts in momentum space are applied. They are not necessary, as can be seen from Fig. 4. After $300 \mathrm{fm} / c \mathrm{nu}-$ cleons with large relative momenta are no longer close together in coordinate space.

The cluster distribution is also not very sensitive to the value chosen for $r_{\min }$. This can also be seen from Table I which shows the exponent $\tau$ for different $r_{\min }$ and different fragment mass bins. At $t=300 \mathrm{fm} / c$ the clusters are well separated in coordinate space as well as in the momentum space due to the Coulomb force.

Figure 5 displays the total mass yield as compared to the experimental data. ${ }^{28}$ Both the theoretical and the experimental mass yield falls off with a power law $A^{-\tau}$,

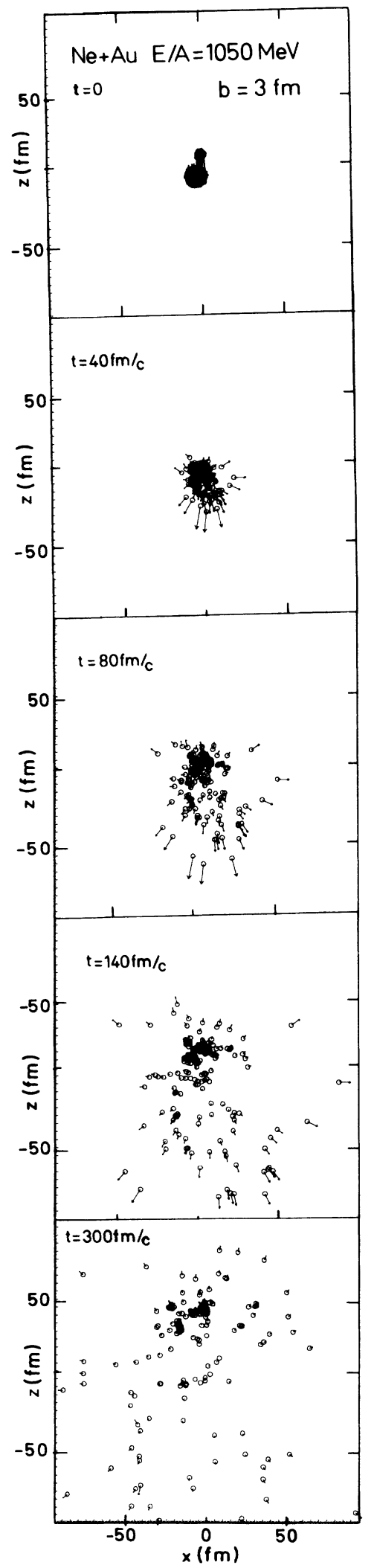

FIG. 4. Time evolution of a collision $1050 \mathrm{MeV} /$ nucleon $\mathrm{Ne}+\mathrm{Au} b=3 \mathrm{fm}$. The projection of all particles onto the $z x$ plane is displayed for five different times: $t=0,40,80,140$, and $300 \mathrm{fm} / c$. The arrows are proportional to the momenta of the particles in the $z x$ plane. The circles have a radius of $1.5 \mathrm{fm}$, so overlapping circles at $t=300 \mathrm{fm} / c$ indicate that the nucleons form a cluster. 


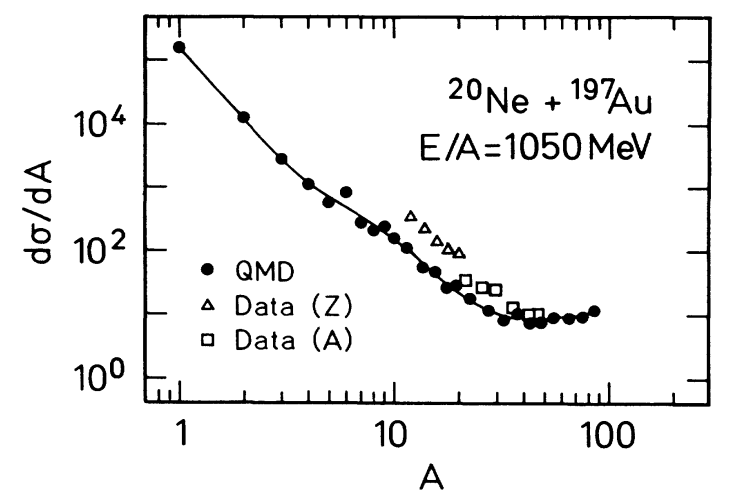

FIG. 5. The inclusive mass yield as compared with experimental data (Ref. 28).

corresponding to a straight line in our double logarithmic plot. For the constant $\tau$ we obtain $\tau=2.44$. The form of the mass yield as well as the value of $\tau: 2<\tau<3$ is consistent with the assumption that the mass yield is a signal of a liquid gas phase transition. This behavior is rather independent of energy and mass number as can be seen by comparison with results of the reaction $\mathrm{Au}(200$ $\mathrm{MeV} /$ nucleon $+\mathrm{Au}){ }^{25}$ The calculated slope of the mass yield curve is close to the slope of the experimental data; however, we underpredict the data by roughly a factor of 2 to 3 . The discrepancy is larger at low masses, which are extracted from the experimental $\sigma(Z)$ under the assumption $\sigma(A)=\frac{1}{2} \times \sigma(Z=A / 2)$ than for those fragments whose masses were directly measured.

This discrepancy is a consequence of the instability of clusters excited with an energy around the particle emission threshold. Whereas - as we have seen - the clusters are stable in their ground state, the higher the excitation, the less correct is our description. Due to a insufficient treatment of the binding energy weakly bound nucleons escape from the excited fragments and hence lower the average cluster mass. Hence our fragments emit 1 or 2 nucleons more than real nuclei with the same excitation energy do. This is a systematic problem which only partially can be counteracted by smaller time steps.

The mass yield has a minimum around $A=50$ and increases again for higher masses. In this particular experiment the mass yield of heavy fragments was not measured. Experiments with similar projectile target combinations show a $U$ shape form of the mass yield, which has a minimum around $A=A_{\text {target }} / 2$ (in absence of fission).

Figure 6 displays (a) the angular distribution and (b) the average fragment velocity as a function of the emis-

TABLE I. $\tau$ parameters for different $r_{\min }$ and for different fragment mass bins $A$.

\begin{tabular}{cccc}
\hline \hline$A$ & $r_{\min }=2 \mathrm{fm}$ & $r_{\min }=3 \mathrm{fm}$ & $r_{\min }=4 \mathrm{fm}$ \\
\hline $1-10$ & 2.86 & 3.07 & 2.99 \\
$1-20$ & 2.68 & 2.82 & 2.83 \\
$1-50$ & 2.33 & 2.44 & 2.50 \\
\hline
\end{tabular}

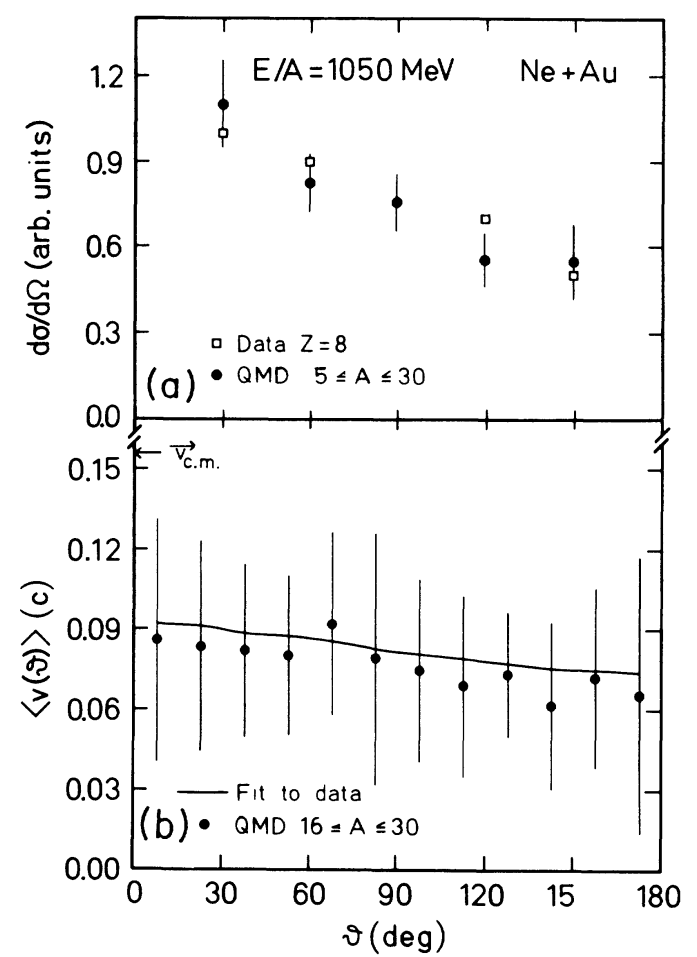

FIG. 6. Angular distribution (a) and average velocity (b) as a function of the emission angle of medium mass fragments. The calculation is compared with data (Ref. 28).

sion angle in the laboratory system. Experimentally we see a decrease in the mass yield by a factor of two from forward to backward angles. This is nicely reproduced by the calculation. As we will see later in detail, this strong dependence rules out an isotropic distribution of fragments in the center-of-mass frame. We observe in Fig. 6(b) only a weak dependence of the average fragment velocity on the emission angle. The average velocity is a little larger than half the center of mass velocity. This means that in the center of mass the fragments are emitted backwards, i.e., the linear momentum transfer to the emitting system is far from being complete. To determine the experimental average velocity we used the fit function (2.3) and the parameters of Table I of Ref. 13. Also for the average fragment velocities we obtain agreement with experiment.

Figure 7(a) displays the number of fast charged particles associated with a fragment of a given size. We applied the experimental cut of a minimal energy of 25 $\mathrm{MeV}$ of the fast particles to our calculation. However, for medium mass fragments our associated multiplicity is larger than seen experimentally. We want to mention that also the experimental value is an extrapolation because the detectors covered only a small part of the total solid angle. Figure $7(\mathrm{~b})$ shows the average velocity of fragments as a function of their size in the laboratory system. We see a very high velocity for low mass particles which gradually decreases for heavier ones. Beyond mass 90, the clusters move backward in the laboratory system. The average momentum transfer to heavy fragments is 


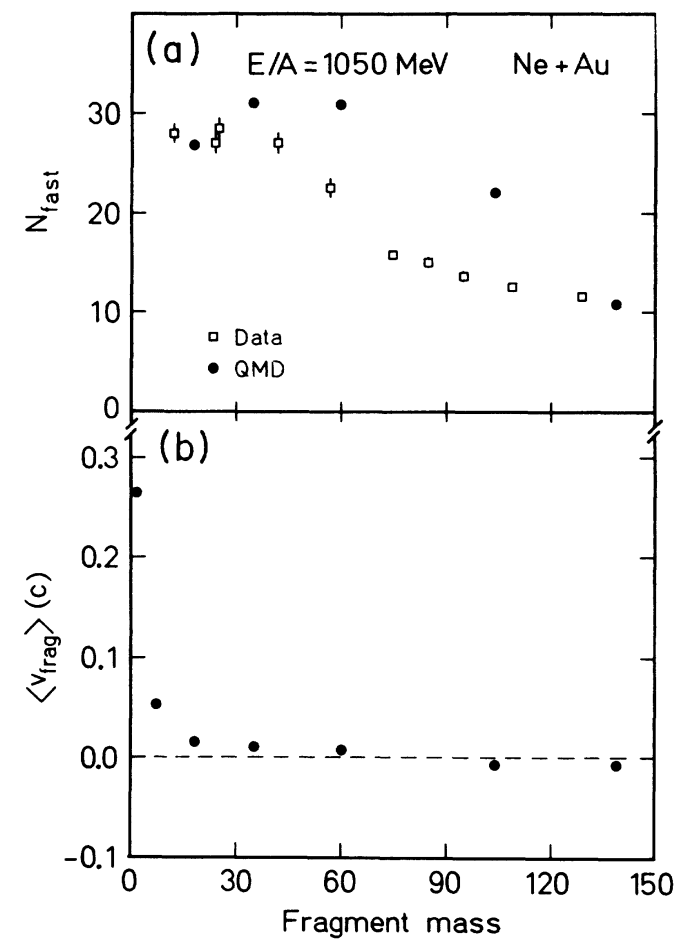

FIG. 7. Average number of fast particles associated with a heavy fragment (a) and the average fragment velocity in the laboratory system (b) as a function of the fragment mass. The data are from Ref. 28.

much smaller than that to the lighter fragments which clearly shows that the fragment of different mass do not result from the same Lorentz frame of reference.

Figure 8 displays the multiplicity distribution of heavy clusters $A \geq 5$. The results of our calculation for ( $b \leq 3 \mathrm{fm}$ ) are compared with data of Refs. 29 and 30 for

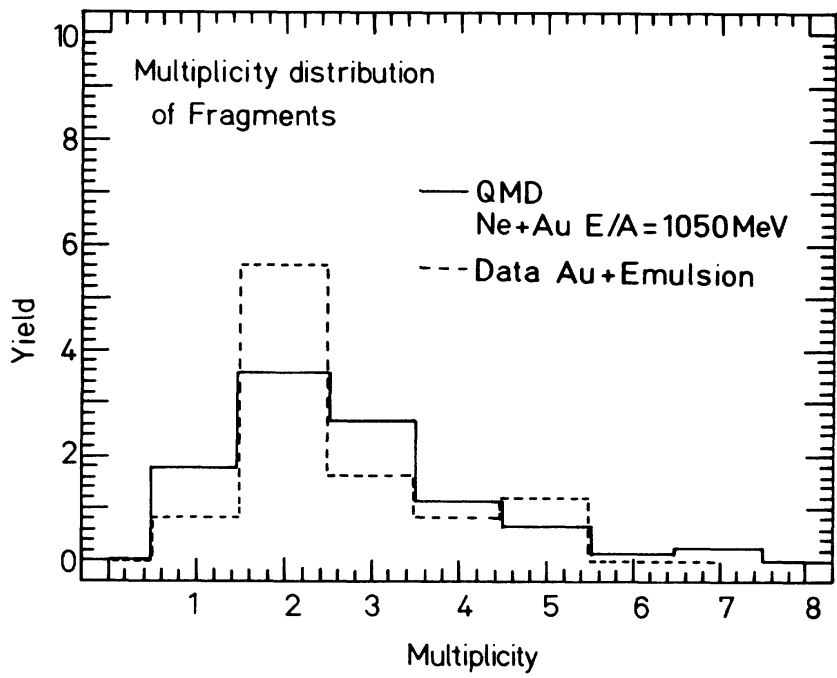

FIG. 8. Multiplicity distribution of heavy clusters $3 \leq Z \leq 20$. The results of our calculation $(b \leq 3 \mathrm{fm})$ are compared with data of Refs. 29 and 30. To allow a comparison we accepted only those experimental events in which no cluster $Z \geq 42$ was observed and the number of target tracks was in between 5 and 8 . By these cuts we want to discard peripheral collisions and those with the heavy emulsion constituents.
$\mathrm{Au}(1 \mathrm{GeV} /$ nucleon $)+$ emulsion. As already mentioned, in this experiment the charges of all $\mathrm{Au}$ fragments were recorded on an event-by-event basis. To allow for a comparison we have selected only those experimental events in which no cluster $42 \leq Z$ was observed and the number of target tracks was in between 5 and 8. By these cuts we want to discard peripheral collisions and those with the heavy emulsion constituents. We see again reasonable agreement with experiment, indicating that on the average several fragments are produced.

\section{B. Predictions and results of the calculation}

\section{Mass yield}

We now proceed and take advantage from the fact that in simulations more information is available than in an experiment. One additional information is the impact parameter dependence of the observed quantities. In Fig. 9 we display the mass yield distribution at different impact parameters. We see a clear impact parameter dependence. At the lowest impact parameter no heavy target remnant survives. The gold nucleus is broken up in many pieces, none of them heavier than $A=80$. At each impact parameter the mass yield is well described by a power law, the slope parameter, however, is vastly different. The most peripheral reactions $(b=7 \mathrm{fm})$ are not violent enough to destroy the target completely. Here less than half of the projectile volume overlaps geometrically with the target. We observe a target remnant of about $A=140$. At the low mass side the mass yield falls off very steeply. Most of these low mass clusters are projectile remnants. There are no clusters with masses $30 \leq A \leq 90$. The intermediate impact parameters show a gradual transition from peripheral to central collisions. The mass of the heavy target remnants decreases but still there is a gap around $A=40$. Also the slope of the mass distribution flattens at small $A$.

From these observations we can immediately draw

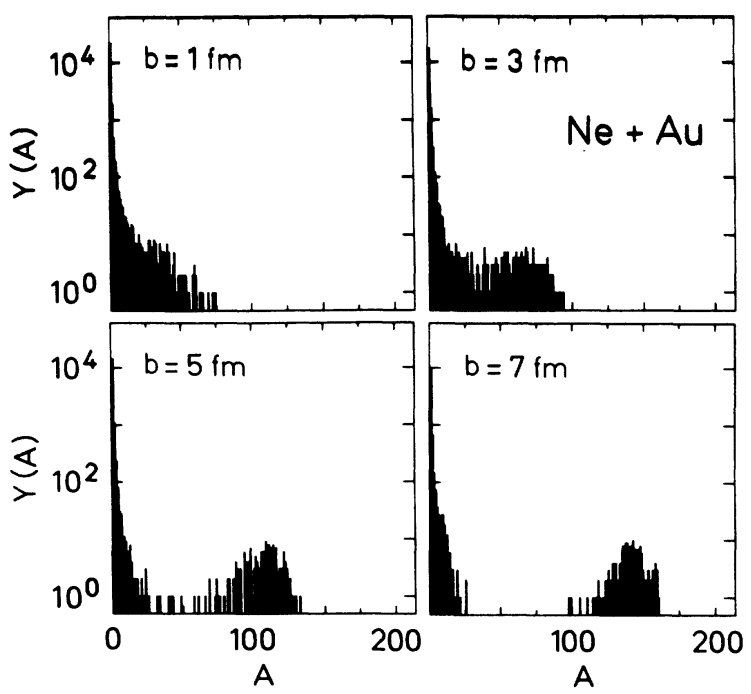

FIG. 9. The mass yield for four different impact parameters $b=1,3,5$, and $7 \mathrm{fm}$ for the reaction $1050 \mathrm{MeV} /$ nucleon $\mathrm{Ne}+\mathrm{Au}$. 
several conclusions.

(a) The power law form of the inclusive mass yield is accidental. It does not reflect a phase transition-which would require a mass yield independent of the impact parameter-but is merely a parametrization of the sum of different forms of mass yields at different impact parameters.

(b) The transition from the power law form to a flat and ultimately increasing mass yield at masses around 40 reflects the different origin of the clusters. Fragments larger than 40 are target remnants. They are produced when the collision is not violent enough to break up the target completely. Their creation is controlled by the impact parameter. Masses around 40 are produced in semicentral collisions by deep spallation. The ultimate increase of the mass yield follows from the increasing probability of peripheral reactions.

(c) The yield of the heaviest cluster provides a tool to determine the impact parameter of the reaction. This method is, according to the calculation, superior in accuracy to the usual method to measure the total multiplicity of light ejectiles.

Figure 10 displays the multiplicity distribution of fragments $A>4$ for the four different impact parameters. We see that at larger impact parameters in most cases the only heavy clusters are the projectile and target remnants. So the average cluster multiplicity is only slightly higher than 2. At more central collisions we observe a larger number of clusters produced. At central collisions the average number of fragments increases to 3.3.

Another point of interest is how the reaction proceeds in time. Here we can prove whether our conjecture that fragments larger than $A=40$ are the end product of a decay chain, whereas the others have a different origin, can be substantiated. This question is addressed in Fig. 11,
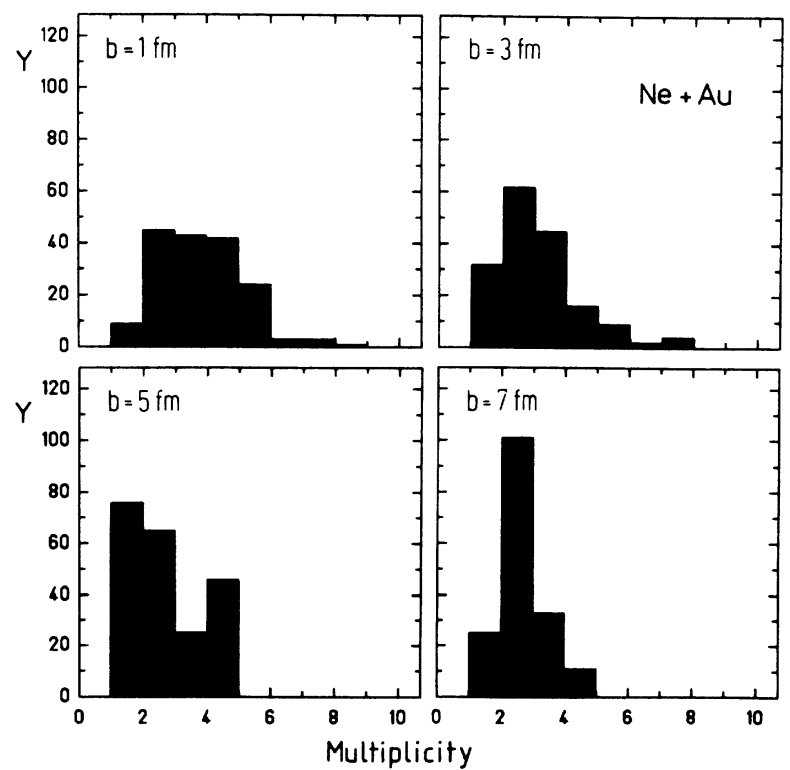

FIG. 10. The multiplicity distribution of fragments heavier than $A=4$ for four different impact parameters $b=1,3,5$, and $7 \mathrm{fm}$ in the reaction $1050 \mathrm{MeV} /$ nucleon $\mathrm{Ne}+\mathrm{Au}$. Please note the different total number of events.

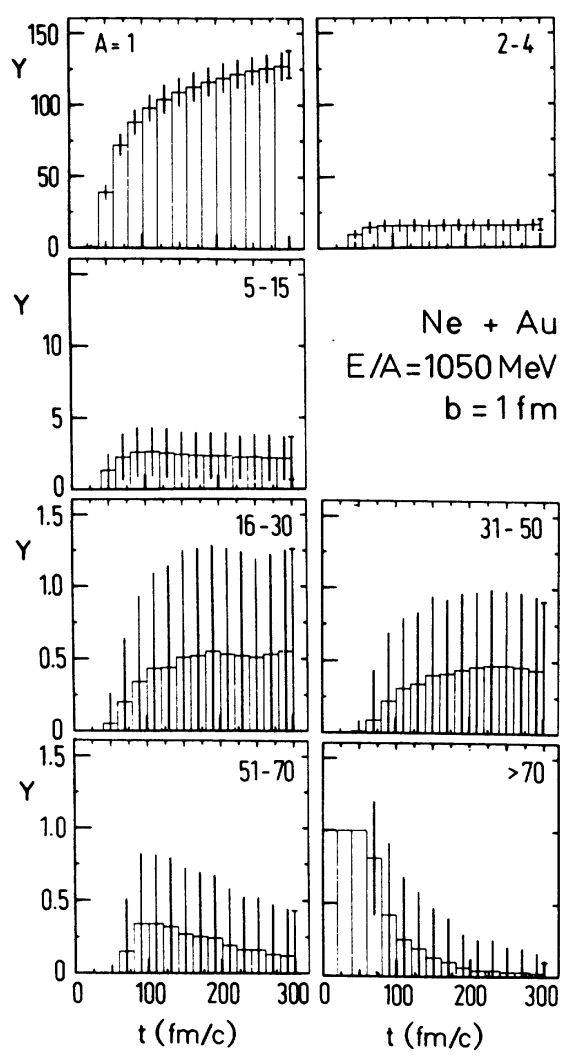

FIG. 11. Time evolution of the fragment number for seven different classes of fragments.

where we display the number of fragments as a function of time for seven mass intervals.

First of all we observe that the mass yield distribution for $A>50$ stabilizes not prior to $300 \mathrm{fm} / c$ $\left(1 \times 10^{-21} \mathrm{sec}\right)$ while for $2<A<30$ the distribution is already stable at $100 \mathrm{fm} / c$. This is a very long time scale for a high energy heavy ion reaction and is in the range of the lifetime of a compound nucleus. Let us first concentrate on the heavy clusters. At $t=50 \mathrm{fm} / c$ the heavy clusters with a mass larger than 70 are not stable but decay successively by emission of nucleons and light clusters. The decay chain can be seen by the subsequent population and depopulation of the different mass bins. Finally, the end products of the decay chain are mostly in the bin $31<A<50$. Along the evaporation chain the clusters emit neutrons and protons and therefore these numbers increase but have almost saturated at $t=300$ $\mathrm{fm} / c$.

The clusters in between $2 \leq A \leq 30$ have a completely different history. They are formed at a very early stage of the reaction and do not get fed from the decaying remnants nor do they decay. After $100 \mathrm{fm} / c$ practically all of them are formed. They emerge from the surface region of the combined system, as we will see, and measure the violence of the reaction being more copiously produced at small impact parameters than at large ones.

Hence, even more pronounced than in the symmetric $\mathrm{Au}(200 \mathrm{MeV} /$ nucleon $)+\mathrm{Au}$ reaction, ${ }^{25}$ we can separate in this asymmetric collision the final clusters into three 
different classes $A=1 ; 2<A<30 ; 30<A$ according to their formation time.

\section{Correlations between initial and final state}

As we have seen, the impact parameter dependence of the mass yield distribution rules out the conclusion that the inclusive data present evidence for a liquid gas phase transition. In this case the mass yield curve would have to be universal because the system always has to come to the critical temperature.

However, the question remains whether equilibrium is reached in the course of the reaction. Since always some fast projectile nucleons emerge from the reaction zone prior to equilibration, the energy available for thermalization may be dependent on the impact parameter and therefore also the mass yield curves may be different. The crucial test for the assumption of complete global thermalization is to check whether the system loses its memory of the initial configuration. If it equilibrates we would expect that the final state particles do not carry any information about the initial state, in particular about their initial position.

The complete recording of the positions and momenta of all particles during the course of the simulation allows to address this question in a direct way. We can immediately investigate correlation between final and initial state which one would expect for a not completely equilibrated system. An obvious candidate for such a correlation is a possible dependence of the probability to find a nucleon finally in a cluster on its initial position. Those nucleons which are in the geometrical overlap of projectile and target supposingly suffer violent collisions more probably. The large momentum transfer then suppresses their probability to find other nucleons with small relative momenta to form a cluster. We study this correlation in Fig. 12. There we distinguish three different classes of nucleons according to the size of the fragments to which they finally belong to (at $t=300 \mathrm{fm} / \mathrm{c}$ ). We investigate the correlation between initial position of the nucleons and the size

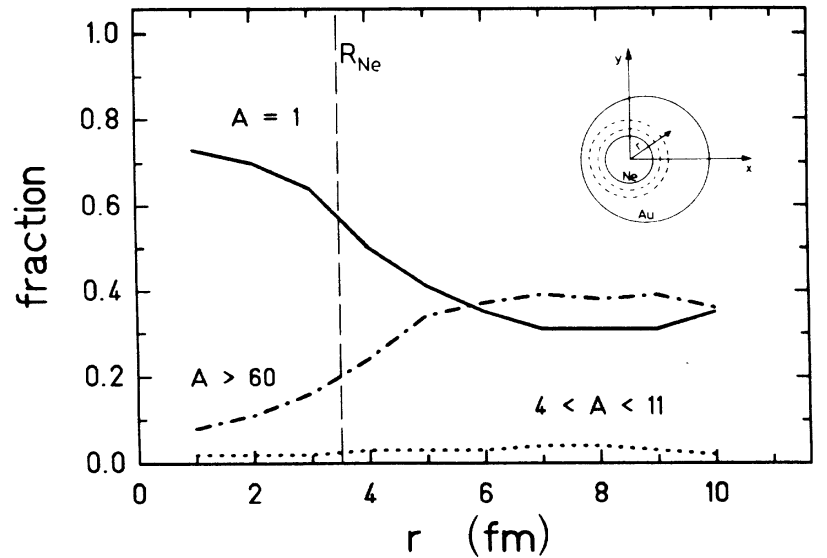

FIG. 12. Correlation between initial and final state of the reaction. We distinguish three classes of nucleons corresponding to the size of the cluster they belong finally $(A=1,4<A<11$, and $A>60$ ) and their relative probability originating from a distance $R$ from the impact point. We display here the fraction $P(r)=N_{i}(r) / \sum N_{i}(r)$ where $N_{i}$ denotes the number of nucleons which end up finally in fragment class $i$. of the clusters they belong to finally. We calculate the distance $R$ in the plane perpendicular to the beam direcon between the initial position of the nucleons and the impact point $\vec{b}$. Then, for a given radius $r$, we calculate the relative probability $P^{A}(r)$ that a nucleon, initially at $r$, ends up finally in a cluster of size $A$. If there were no correlations, this probability should be independent of $r$. We see, however, strong correlations for protons and neutrons as well as for heavy clusters. Nucleons in the overlap region between projectile and target have a much higher probability to end up as individual protons and neutrons than to be part of a cluster. Clusters mainly consist of spectator matter (those parts of projectile and target which do not overlap). Since the fragments are excited when formed, they have to emit nucleons. Therefore, we observe also protons and neutrons which were originally located quite far from the impact point of the projectile. Medium mass clusters, as we can see, are formed from nucleons all over the place without a significant preference. From the observed correlations we can conclude that in the course of the interaction the system does not reach a global equilibration as assumed in a number of model calculations. ${ }^{7,8,15,16}$

Part of this correlation one would expect from the naive fireball ${ }^{43}$ or firestreak ${ }^{44}$ model in which the geometrically overlapping nucleons equilibrate. They are surrounded by cold spectator matter, and there is no communication between both regions. This model limits the initial position of nucleons, which are finally observed as protons or neutrons, to $r<R_{\mathrm{Ne}}$ whereas those nucleons, which are finally contained in clusters, are initially at $r>R_{\mathrm{Ne}}$. However, we do not find such a clear cut and these models can also not account for the origin of the medium mass clusters.

\section{Momentum space distribution}

In principle the present theory can predict the triple differential cross section $d^{3} \sigma / d E d \Omega d A$. However, due to computational expenditure we restrict ourselves to mean values for the time being.

We start with the laboratory double differential cross section $d^{2} \sigma / d y d p_{t}$, displayed in Fig. 13, for different classes of fragments and two different impact parameters. The contour lines are separated by a factor of 2 . We see that light fragments have a highly nonisotropic emission pattern even in central collisions. There are many fast particles in forward direction which are not counterbalanced at backward angles. These particles have a finite emission angle whose origin will be investigated in the next section. The emission pattern of medium mass clusters is to $10 \%$ not isotropic as experimentally observed by Warwick et $a .^{28}$ who have found that the double differential cross section of medium mass clusters cannot be described by a single thermal source which emits fragments isotropically in its rest system. Comparing $d \sigma / d \Omega d E d Z$ at different laboratory angles one has two possibilities to define the source velocity: either (a) one requires that the slope of the energy distribution (i.e., the "temperature") does not depend on the emission angle in the rest system or (b) one assumes that the Coulomb peak appears at the same energy in the rest system. If there 


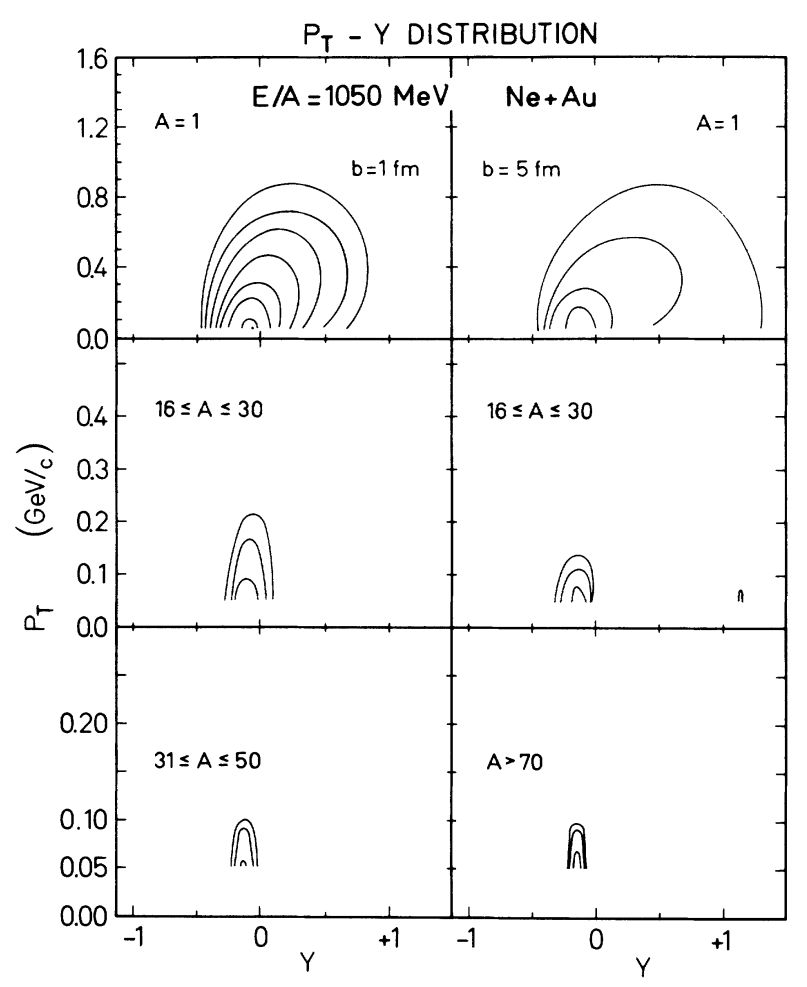

FIG. 13. Contour plot of the double differential cross section $d^{2} \sigma / d p_{t} d y$ for two impact parameters $(b=1$ and $5 \mathrm{fm})$ and three different mass intervals for the reaction 1050 $\mathrm{MeV} /$ nucleon $\mathrm{Ne}+\mathrm{Au}$.

were an isotropic emission of a single source both methods would coincide. The experiment shows differences between both methods. Applying the second method it seems that the fragments have gained additional momentum in beam direction, i.e., show a higher "temperature." This gives rise to an elliptical emission pattern in the rest frame of the source. The heavier clusters, on the contrary, exhibit an isotropic emission pattern in their rest system which moves with is close to zero velocity in the laboratory system. Note the different scales of the transverse momentum.

Figure 14 presents the rapidity distribution of the different classes of fragments at two impact parameters. It is quite obvious that the rapidity distributions are not thermal. This is in contrast to the results for central collisions in the symmetric $\mathrm{Au}(200 \mathrm{MeV} /$ nucleon $)+\mathrm{Au}$ collisions. ${ }^{25}$ There the particle distribution in very central collisions $(b=1 \mathrm{fm})$ can be described by one single stopped midrapidity source. For the large impact parameter $(b=7 \mathrm{fm})$ we see clearly a projectile and a target region. For low impact parameters the projectile dived into the target and is strongly slowed down. Once more we can see that the medium mass clusters do not come from a single well defined rest system.

Figure 15 finally shows the transverse "temperature," i.e., up to a constant the second moment of the transverse momentum distribution,

$$
\Delta=m / 3\langle v-\bar{v}\rangle^{2} \frac{\cos (\theta)}{\cos (\theta)-\cos ^{3}(\theta) / 3},
$$
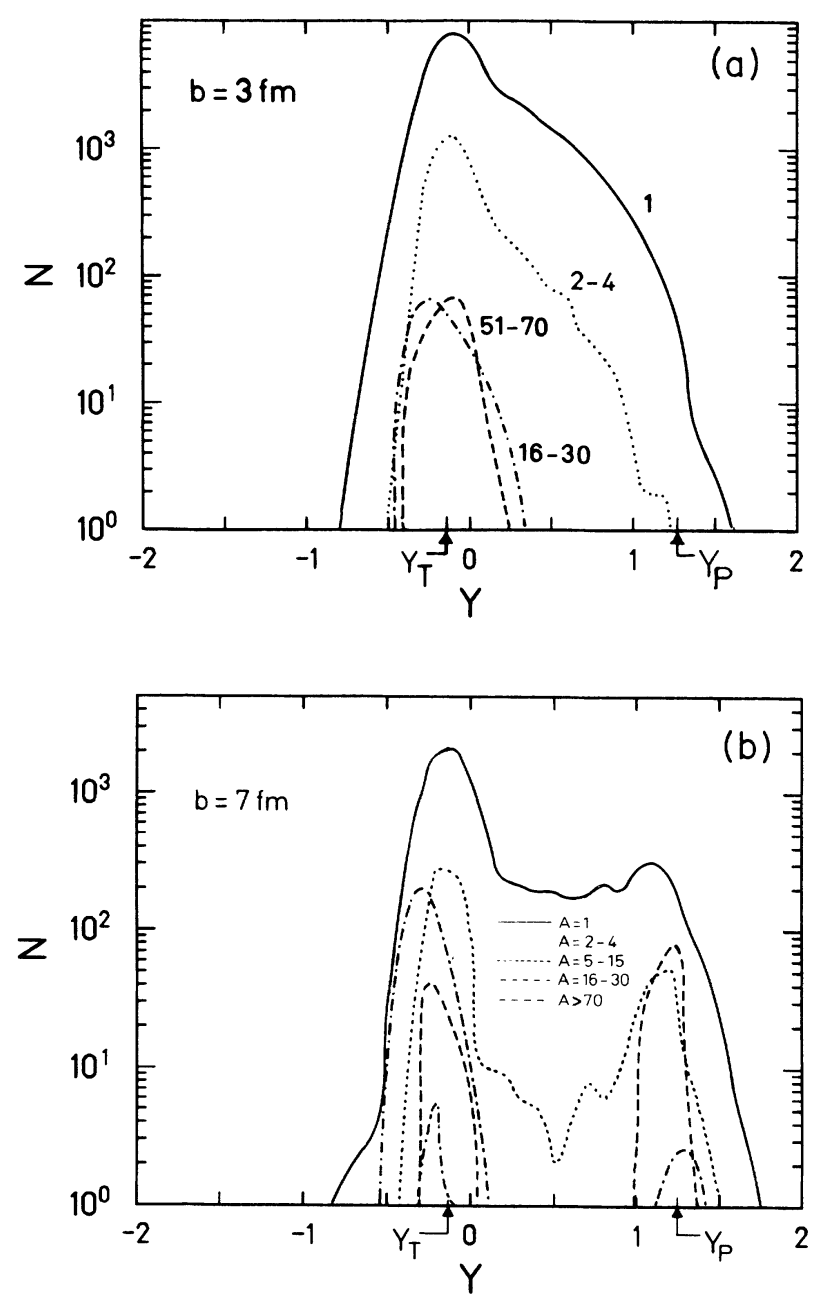

FIG. 14. Rapidity distribution of different mass intervals for $b=3$ and $7 \mathrm{fm}$ of the reaction $1050 \mathrm{MeV} /$ nucleon $\mathrm{Ne}+\mathrm{Au}$.

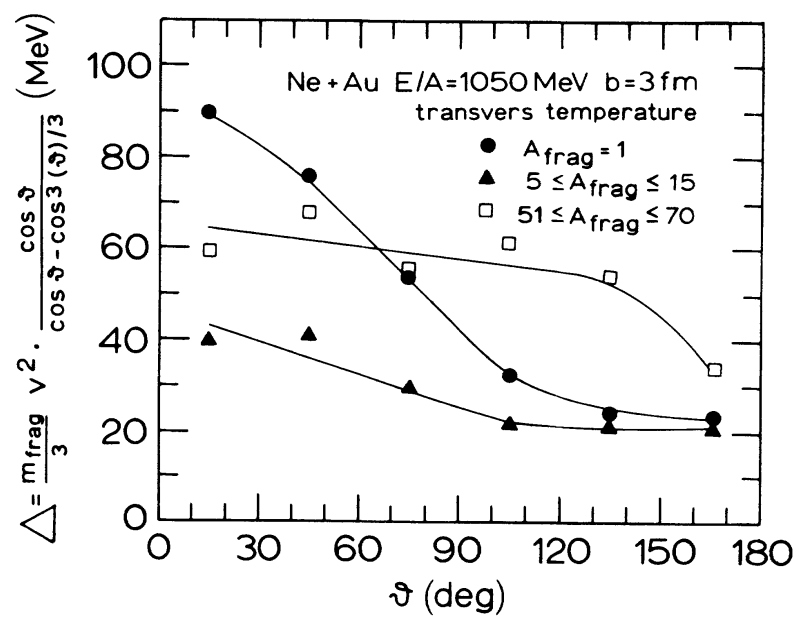

FIG. 15. Transverse "temperature" $\Delta=m / 3<v^{\text {frag }}$ $\left.-\bar{v}^{\text {frag }}\right\rangle^{2} \cos (\theta) /\left[\cos (\theta)-\cos ^{3}(\theta) / 3\right]$ of fragments for different mass intervals. 
for different fragment classes in their rest system as a function of the emission angle. For a system which is also longitudinal equilibrated this quantity coincides with the apparent temperature. Isotropic emission would result in an angle independent $\Delta$. We see, however, an enhancement of about a factor of 2-6 in forward direction as compared to the backward direction, for low mass fragment masses. Only the largest mass bin, which has also the lowest statistics, can be considered as isotropically emitted. Also the average $\Delta$ of different clusters are different. $\Delta=30.1,27.3,57.4 \mathrm{MeV}$ for the mass bin $A=1,5 \leq A \leq 15,51 \leq A \leq 70$.

From all of these observations we conclude that the momentum space distribution of the different cluster classes are widely different. A single source cannot be identified, rather each class of clusters seems to stem from a continuous source distribution.

\section{What cases fragmentation?}

There remains the question of what actually causes the fragment formation. We have seen that clusters are not formed in a globally equilibrated environment. Hence processes other than statistical decay have to be taken into account. We have seen that the medium mass clusters are emitted from the system already quite early, long before the target evaporation chain ceases. In this section we investigate the details of this process. Since clusters are produced by fluctuations of the system, we have to investigate the cluster formation on an event-by-event basis, looking for the specific environment around a prefragment, i.e., those nucleons which finally form the fragment or are emitted from the fragment, in a given simulation of the reaction.

We start out by examining how projectile and target interact at the beginning of the interaction. Figure 16 displays the density profile of the system for central collisions from $10-35 \mathrm{fm} / c$ in steps of $5 \mathrm{fm} / c$. In Fig. 17 we see the velocity profile, separately for those nucleons initially belonging to projectile respective target from 10-20 $\mathrm{fm} / c$ in steps of $5 \mathrm{fm} / c$. Arrows are only plotted if the local density is larger than $0.1 \rho_{0}$. We have averaged in this figure over ten events, so fluctuations are reduced, but not completely washed out.

At $10 \mathrm{fm} / c$ the projectile has completely dived into the target. The projectile velocity is much faster than the sound velocity of nuclear matter. Therefore the time scale for the transverse expansion of the projectile is small as compared to the time scale for the projectile to transverse the target. The root mean square radius of the projectile nucleons has not increased up to $15 \mathrm{fm} / c$. The peak compression has increased to $2.1 \rho_{0}$. While the projectile nucleons travel through the target they experience strong transverse forces due to the strong density gradient at the surface of the projectile. Therefore they pick up transverse velocity and are deflected to finite angles. A shock profile develops which moves inwards into the projectile nucleus because the outer nucleons have already been carried away by the sidewards traveling compression wave. The source itself decelerates gradually but has still supersonic velocity. In an infinite system this is the situation where a Mach cone would be formed and indeed the form of the velocity distribution at 20 $\mathrm{fm} / c$ resembles very much such a velocity profile. However, one has to keep in mind that we have only a system

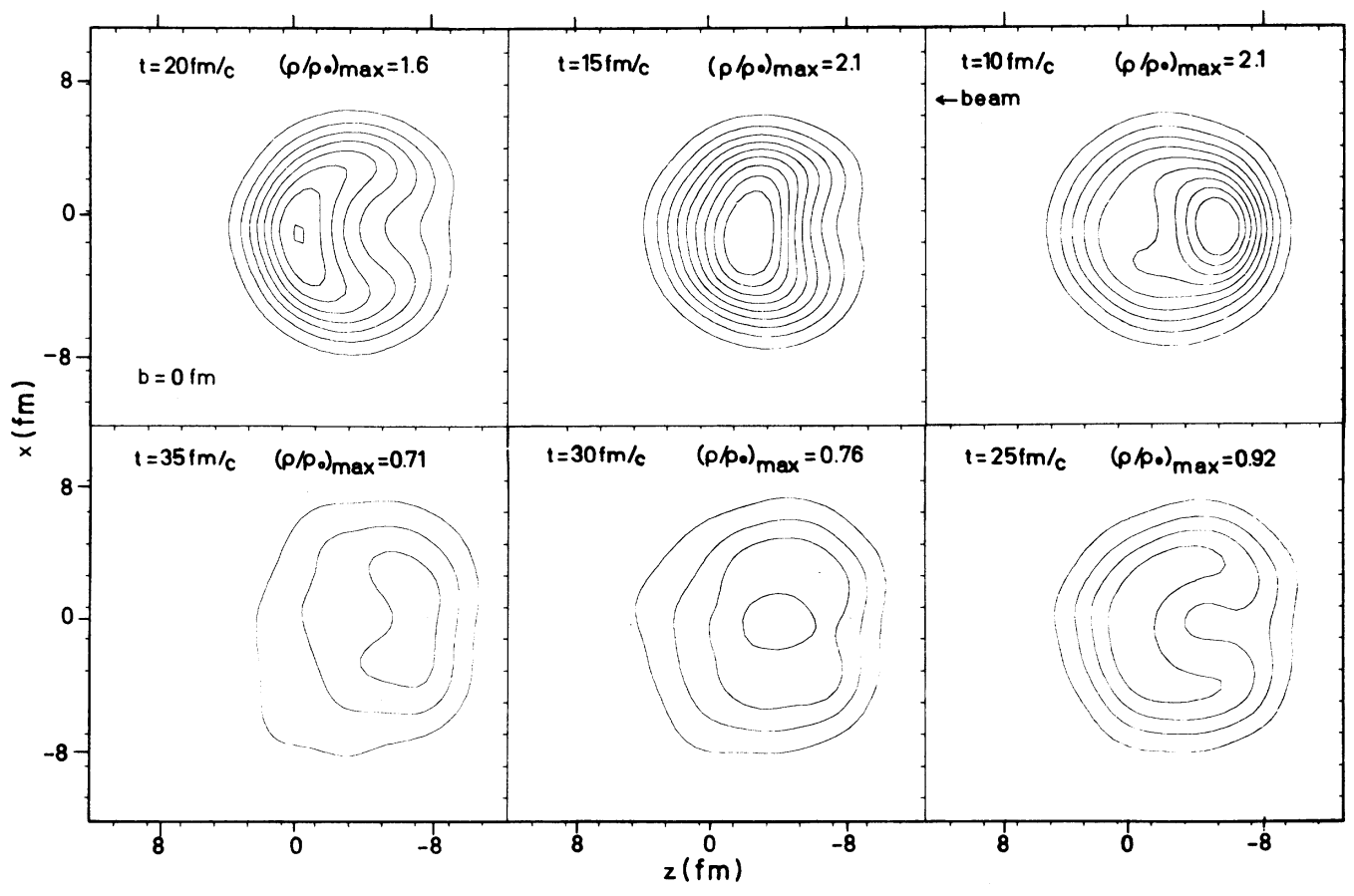

FIG. 16. Density profile in the $z x$ plane at the beginning of the reaction, $t=10-35 \mathrm{fm} / c$, in steps of $5 \mathrm{fm} / c$. The contour lines are separated by a factor of 2 , starting with $\rho / \rho_{0}=0.2$. We display a central collision $\mathrm{Ne}(1050 \mathrm{MeV} / \mathrm{nucleon})+\mathrm{Au}$. 


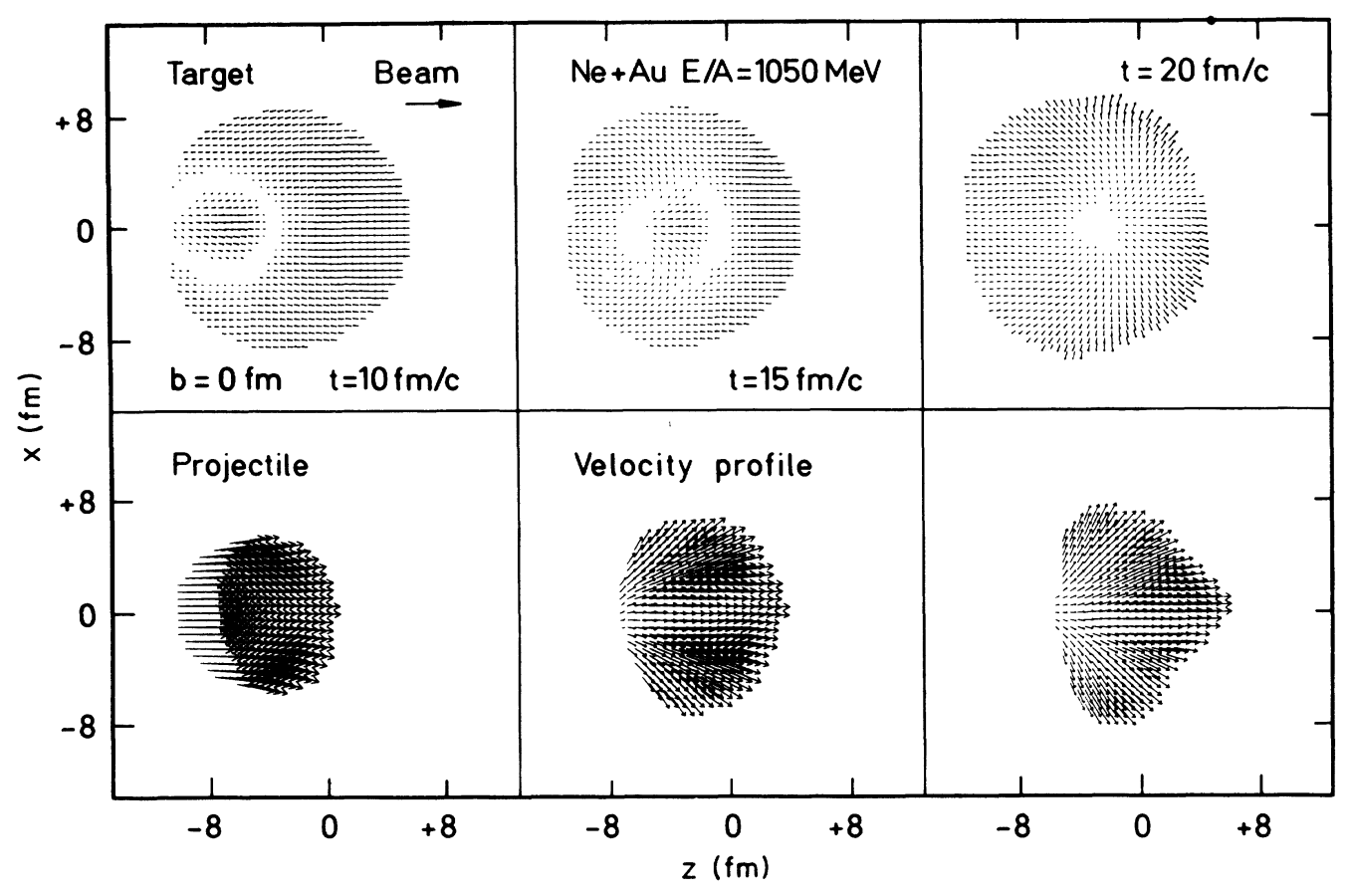

FIG. 17. Velocity profile for $b=0 \mathrm{fm}$ in the $z x$ plane at the beginning of the reaction, $t=10-20 \mathrm{fm} / c$, in steps of $5 \mathrm{fm} / c$, separately displayed for projectile and target nucleons. The length and direction of the arrows are proportional to the velocity in the $z x$ plane. An arrow is only drawn if the density is larger than $0.1 \rho_{0}$.

of 217 particles and therefore, we do not obtain a sharp discontinuity in the density as in systems with a macroscopic particle number. Behind the cone we observe a rarefaction region which heels, however, due to target surface nucleons streaming inwards. By $35 \mathrm{fm} / c$ this rarified region is filled and has again the highest density. Whereas, at the beginning projectile and target interpenetrate (the density provides a high Pauli blocking rate), finally the projectile drags along some target nucleons. So at $20 \mathrm{fm} / c$ we see that in forward direction the target nucleons having the same direction of motion as the projectile nucleons, whereas in backward direction they move quite collectively with roughly half of the center of mass momentum. This time evolution of the reaction is close to that predicted by hydrodynamical calculations. $^{42}$

How a prefragment, i.e., a particle unstable fragment, experiences this situation is displayed in Fig. 18: Figure 18(a) shows the time evolution of mean values of different quantities for a large prefragment $(A=24)$. This prefragment emits still few nucleons before it is finally (at $t=300 \mathrm{fm} / \mathrm{c}$ ) registered as a fragment. Figure $18(\mathrm{~b})$ shows the analogous quantities for a small fragment $(A=6)$. The upper picture displays the number of collisions per fragment nucleon. Initially, i.e., before the projectile reaches nucleons of the fragment, no collisions occur. Between the arrival of the projectile and the separation of the fragment from the remnant we observe a quite high collision rate. When the fragment is formed there is still excitation energy which allows further collisions among the fragment nucleons. The next graph shows the density
$\rho=\frac{1}{A_{\mathrm{frag}}} \sum_{j \in A_{\mathrm{frag}}} \sum_{i \in A}\left(\frac{1}{2 \pi L}\right]^{3 / 2} \exp -\left[\mathbf{R}_{\mathrm{j}}-\mathbf{R}_{\mathrm{i}}(t)\right]^{2} / 2 L$

in units of $\rho_{0}$ and the average radial force

$$
F_{\text {rad }}=\frac{1}{A_{\text {frag }}} \sum_{j \in A_{\text {frag }}} \frac{\mathbf{F}_{\mathrm{j}}\left(\mathbf{r}_{\mathrm{j}}-\mathbf{r}_{\text {c.m. target }}\right)}{\left|r_{j}-r_{\text {c.m. target }}\right|} .
$$

Radial means here with respect to the center of mass of the target. As already expected from the previous figure we see a strong increase in the density when the projectile matter hits the nucleons which will form the fragment. Initially the collective momentum of those nucleons points inwards. The density, however, causes a strong radial repulsive force which reverses the direction of the average momentum

$$
p_{\text {rad }}=\frac{1}{A_{\text {frag }}} \sum_{j \in A_{\text {frag }}} \frac{\mathbf{p}_{\mathbf{j}}\left(\mathbf{r}_{\mathbf{j}}-\mathbf{r}_{\mathrm{c} . \mathrm{m} . \text { target }}\right)}{\left|\left(r_{j}-r_{\text {c.m. target }}\right)\right|} .
$$

This is displayed in the next row.

So far it seems that collisions are not required at all to cause fragments to break off. This, however, is not true. If the $n-n$ collisions are suppressed we do not find fragmentation. Collisions have a twofold influence on the fragmentation processes. Firstly, they decelerate the projectile nucleons and hence increase the density in the projectile region. Secondly, they may provide an additional momentum transfer to those nucleons which are going to form a fragment. To check whether the second mechanism is important, we compare the actual average radial 


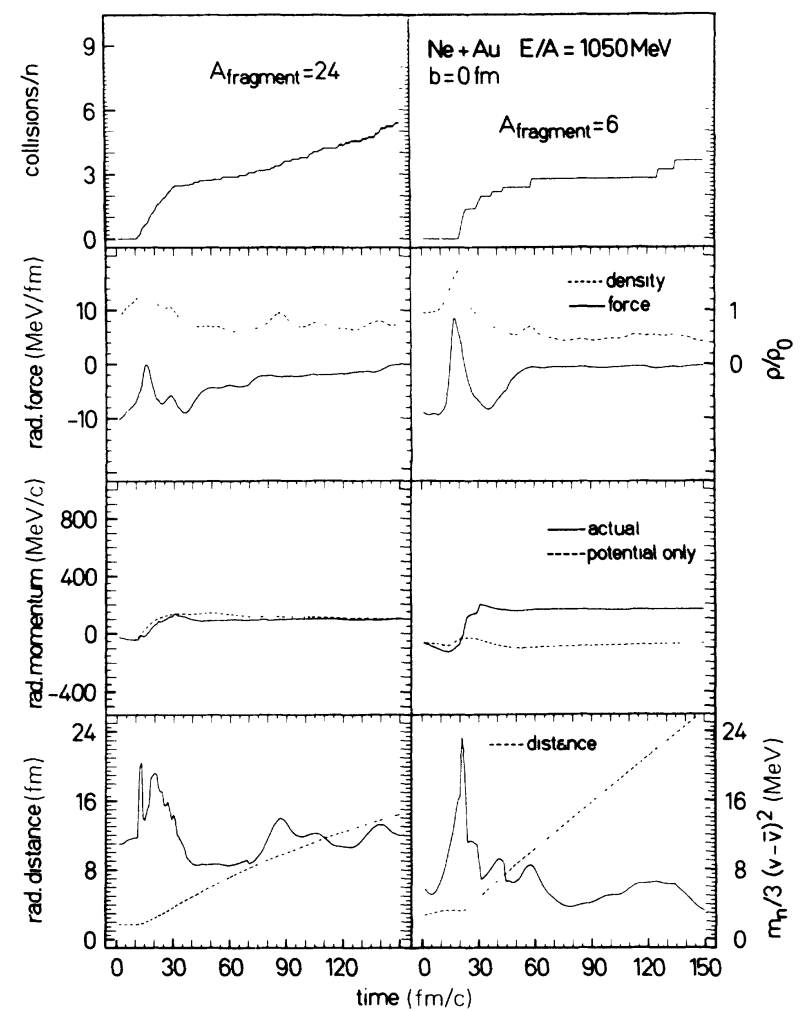

FIG. 18. Time evolution of a single prefragment produced in the reaction $1050 \mathrm{MeV} /$ nucleon $\mathrm{Ne}+\mathrm{Au} b=0 \mathrm{fm}$. The properties of a heavy $(A=24)$ prefragment are displayed in (a), those of a light prefragment $(A=6)$ in (b). We show the average number of collisions, the mean radial force, the density, the mean radial momentum, the mean radial distance from the center of the target, and the "temperature" $\Delta=(m / 3)\langle v-\bar{v}\rangle^{2}$ as a function of time. The values displayed are averaged over the fragment constituents. For details we refer to the text.

momentum with that caused by the average radial field only. The latter is determined by

$$
p_{\text {rad }}^{\text {field }}(t)=\int_{0}^{t} F_{\text {rad }}\left(t^{\prime}\right) d t^{\prime}+p_{\text {rad }}(t=0) .
$$

The difference between both reveals the importance of the momentum transfer to the fragment nucleons due to collisions. The results are displayed in the same graph. In the case of the large cluster the final momenta are almost identical, whereas the small cluster would not be broken off at all (it still would have inward directed radial momentum). So the role of collisions for the actual breakup process is ambiguous.

The lower graph displays the time evolution of the internal excitation of the fragment. We define a "temperature"

$$
\Delta=\frac{1}{A_{\text {frag }}} \sum_{j \in A_{\text {frag }}} \frac{m}{3}\left\langle v_{j}-v_{a v}\right\rangle^{2} .
$$

Keep in mind, however, that this is not a true temperature since it also includes the Fermi momentum. We see only a small increase in the course of the reaction. So the prefragments are only moderately excited and there seems to be no equilibration between the internal degrees of freedom and the translational motion. This is in agreement with recent experiments, which show that the excitation of prefragments corresponds to temperature of 5 $\mathrm{MeV}$, independent of the beam energy. ${ }^{45}$

The velocity profile of the same reaction for $b=6 \mathrm{fm}$ is displayed in Fig. 19. Here the projectile gets little deflected but still drags along some target nucleons. The target gets only little excited and most of the target nucleons retain their initial velocity up to $25 \mathrm{fm} / c$, when the interaction between projectile and target is over. Only close to the interaction zone we see a disturbance in the velocity field which ultimately leads to an excitation of the target. Since particles from this excited zone which travel towards the center of the target have a higher chance to transfer their momentum to other target nucleons than those traveling to the surface of the target, we observe a transverse momentum transfer to the target in $-x$ direction which is compensated by the momentum of the emitted single particles in the opposite direction.

\section{CONCLUSION}

We have presented a microscopic dynamical $N$-body approach to heavy ion collisions which consistently incorporates the formation of clusters. Heavy ion reactions can now be studied theoretically on an event-by-event basis from the initial configuration to the final distribution of nucleons and fragments in phase space. This is achieved by following the time evolution of the $N$-body system which interacts via mutual two and three body potentials and a residual interaction with the Pauli blocked nucleon nucleon scattering cross section.

In this paper we reported on simulations of the reaction $1050 \mathrm{MeV} /$ nucleon $\mathrm{Ne}+\mathrm{Au}$. Our results agree well with the available data, in particular with the experimental slope, the absolute value of the mass yield curve, the angular distributions, the mean velocities of different fragments, and with the experimental multiplicity distribution. We do not find a single source which emits particles isotropically, which is consistent with the experimental data.

We find a strong impact parameter dependence of the mass yield curve which rules out the conjecture that the power law form of the inclusive mass yield curve provides a signature that the system is close to the critical point of a liquid gas phase transition. The agreement of the inclusive mass yield distribution with a form expected from a system close to its critical point is purely accidental.

We observe furthermore strong geometric correlations between the entrance and exit channel of the reaction. Nucleons retain information about their initial position. This rules out the assumption that the system equilibrates globally to a compoundlike nucleus in the reaction. If that were true any information about the initial state would be lost. Consequently, a single source thermodynamical approach to multifragmentation in heavy ion reactions cannot be considered as adequate.

The emission of medium mass fragments $2 \leq A \leq 40$ occurs in the very early high density stage of the reaction only. At later times a compoundlike remnant is formed which emits protons and neutrons. The decay chain of 


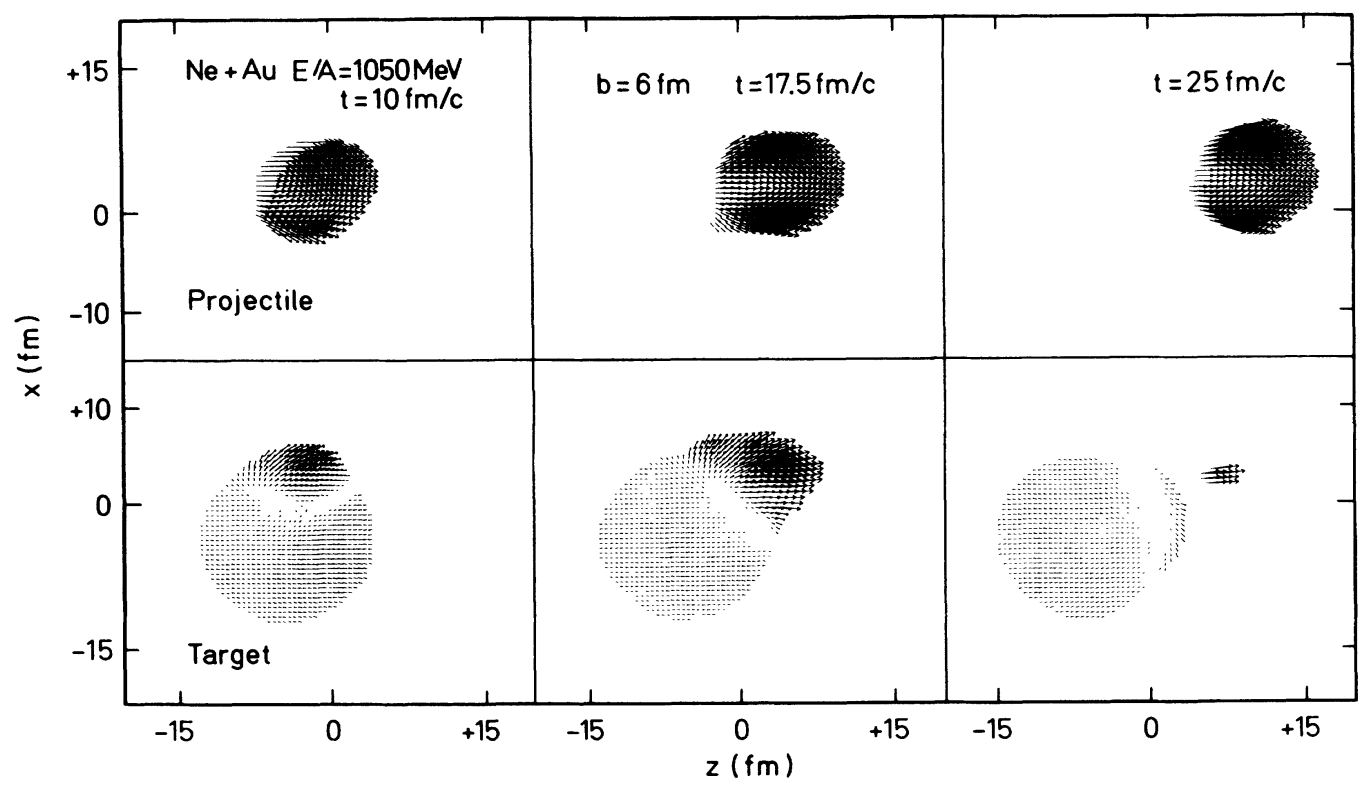

FIG. 19. Velocity profile for $b=6 \mathrm{fm}$ in the $z x$ plane at the beginning of the reaction, $t=10-20 \mathrm{fm} / c$, in steps of $5 \mathrm{fm} / c$, separately displayed for projectile and target nucleons. The length and direction of the arrows are proportional to the velocity in the $z x$ plane.

these target remnants yield fragments with masses down to $A=40$. Fragments $2 \leq A \leq 30$ have a completely different origin. They are not evaporation residues but stem from the surface of the system. They separate from the rest system already at a very early time when the density is highest. They measure the violence of the reaction, i.e., the number of these clusters decreases strongly as a function of impact parameter. The medium mass clusters are kicked off from the system as a result of the high density wave and its repulsive force caused by the interpenetrating projectile. The clusters are only moderately excited initially and later emit only few nu- cleons before being detected. The intermediate mass clusters can therefore serve as a unique tool to study the compression stage of the reaction. This will be the focus of a subsequent paper.

\section{ACKNOWLEDGMENTS}

We want to thank Prof. J Hüfner for many fruitful discussions and critical comments. This work has been funded in part by the German Federal Minister for Research and Technology (BMFT) under Contract Nos. 06-HD-776, 06-0F-772, and by the Gesellschaft für Schwerionenforschung (GSI).
${ }^{1}$ J. E. Finn et al., Phys. Rev. Lett. 49, 1321 (1982).

${ }^{2}$ M. E. Fisher, Physics 3, 255 (1967).

${ }^{3}$ A. D. Panagiotou, M. W. Curtin, and D. K. Scott, Phys. Rev. C 31, 55 (1982).

${ }^{4}$ G. Fai and J. Randrup, Nucl. Phys. A404, 551 (1983).

${ }^{5}$ B. V. Jacak et al., Phys. Rev. Lett. 51, 1846 (1983); Phys. Rev. C 35, 1751 (1987).

${ }^{6}$ H. Stöcker et al., Nucl. Phys. A400, 63c (1983).

${ }^{7}$ D. H. E. Gross and Ciao-ze Zhang, Phys. Lett. 161B, 47 (1985).

${ }^{8}$ J. P. Bondorf, R. Donangelo, I. N. Mishustin, C. J. Petrick, H. Schulz, and K. Sneppen, Nucl. Phys. A443, 347 (1985); J. P. Bondorf, R. Donangelo, I. N. Mishustin, and H. Schulz, ibid. A444, 460 (1985).

${ }^{9}$ W. A. Friedman and W. G. Lynch, Phys. Rev. C 28, 950 (1983).

${ }^{10}$ X. Campi, J. Desbois, and E. Lipparini, Phys. Lett. 142B, 8 (1984).

${ }^{11}$ W. Bauer, D. R. Dean, U. Mosel, and U. Post, Phys. Lett.
150B, 53 (1985).

12J. Aichelin and J. Hüfner, Phys. Lett. 136B, 15 (1984).

13J. Aichelin, J. Hüfner, and R. Ibarra, Phys. Rev. C 30, 107 (1984).

14J. Hüfner, Phys. Rep. 125, 129 (1985).

${ }^{15}$ B. Strack and J. Knoll, Z. Phys. A 315, 249 (1984); J. Knoll and B. Strack, Phys. Lett. 149B, 45 (1984).

${ }^{16}$ R. J. Lenk and V. R. Pandharipande, Phys. Rev. C 34, 177 (1986), and references therein.

${ }^{17}$ J. Aichelin and G. Bertsch, Phys. Rev. C 31, 1730 (1985); G. Bertsch, S. Das Gupta, and H. Kruse, ibid. 29, 673 (1984).

${ }^{18}$ H. Kruse, B. V. Jacak, and H. Stöcker, Phys. Rev. Lett. 54, 289 (1985); Phys. Rev. C 31, 1770 (1985); H. Stöcker and W. Greiner, Phys. Rep. 137, 277 (1986), and references therein.

${ }^{19}$ C. Gregoire, B. Remaud, F. Sebille, L. Vinet, and Y. Raffray, Nucl. Phys. A465, 317 (1987), and references therein.

20J. Aichelin and E. Remler, Phys. Rev. C 35, 1291 (1987).

${ }^{21}$ J. Aichelin and C. M. Ko, Phys. Rev. Lett. 55, 2661 (1985). 
${ }^{22}$ W. Bauer, G. Bertsch, and S. Das Gupta, Phys. Rev. Lett. 58, 863 (1987).

${ }^{23}$ S. Das Gupta, C. Gale, J. Gallego, H. H. Gan, and R. D. Ratna Raju, Phys. Rev. C 35, 556 (1987).

${ }^{24}$ J. Aichelin and H. Stöcker, Phys. Lett. B 176, 14 (1986).

${ }^{25}$ G. Peilert, A. Rosenhauer, H. Stöcker, W. Greiner, and J. Aichelin, Mod. Phys. Lett. (in press), G. Peilert, diploma thesis, University of Frankfurt, 1988 (unpublished); A. Rosenhauer et al., J. Phys. (Paris) Colloq. C4, 395 (1986).

${ }^{26}$ A. Rosenhauer, G. Peilert, H. Stöcker, W. Greiner, and J. Aichelin, Phys. Rev. C (in press); A. Rosenhauer, thesis, Gesellschaft für Schwerionenforschung, 1988 (Gesellschaft Report GSI-88-09, 1988).

${ }^{27}$ G. E. Beauvais, D. H. Boal, and J. C. Wong, Phys. Rev. C 35, 545 (1987).

${ }^{28}$ A. I. Warwick, H. H. Wiemann, H. H. Gutbrod, M. R. Maier J. Peter, H. G. Ritter, H. Stelzer, and F. Weik, Phys. Rev. C 27, 1083 (1983); A. I. Warwick, private communication.

${ }^{29}$ C. J. Waddington and P. S. Freier, Phys. Rev. C 31, 888 (1985).

30J. Aichelin and X. Campi, Phys. Rev. C 34, 1643 (1986).

${ }^{31}$ A. R. Bodmer and C. N. Panos, Phys. Rev. C 15, 1342 (1977).

${ }^{32}$ L. Wilets, Y. Yariv, and R. Chestnur, Nucl. Phys. A282, 359 (1977).
${ }^{33}$ J. Molitoris, J. B. Hoffer, H. Kruse, and H. Stöcker, Phys. Rev. Lett. 53, 899 (1984).

${ }^{34}$ S. Jones and E. Remler, Ann. Phys. (NY) 180, 152 (1987).

35J. Aichelin and H. Stöcker, Phys. Lett. 163, 59 (1985).

${ }^{36}$ P. Carruthers and M. Zachariasen, Rev. Mod. Phys. 55, 245 (1983).

${ }^{37}$ J. Aichelin, G. Peilert, A. Rosenhauer, H. Stöcker, and W. Greiner, Phys. Rev. 58, 1926 (1987).

${ }^{38}$ K. T. R. Davis and S. E. Koonin, Phys. Rev. C 23, 2042 (1981).

${ }^{39}$ J. P. Jeukenne, A. Lejeune, and C. Mahaux, Phys. Rep. 25, 83 (1976).

${ }^{40}$ B. terHaar and R. Malfliet, Phys. Rep. 149, 207 (1987).

${ }^{41}$ J. Cugnon, T. Mizutani, and J. Vandermeulen, Nucl. Phys. A352, 505 (1981).

${ }^{42}$ H. Stöcker, J. A. Maruhn, and W. Greiner, Phys. Rev. Lett. 44, 725 (1980).

${ }^{43}$ G. Westfall, J. Gosset, P. J. Johanson, A. M. Poskanzer, W. G. Meyer, H. H. Gutbrod, A. Sandoval, and R. Stock, Phys. Rev. Lett. 37, 1202 (1976).

${ }^{44}$ W. D. Myers, Nucl. Phys. A296, 177 (1978).

45J. Pochodzalla et al., Phys. Rev. C 35, 1695 (1987). 


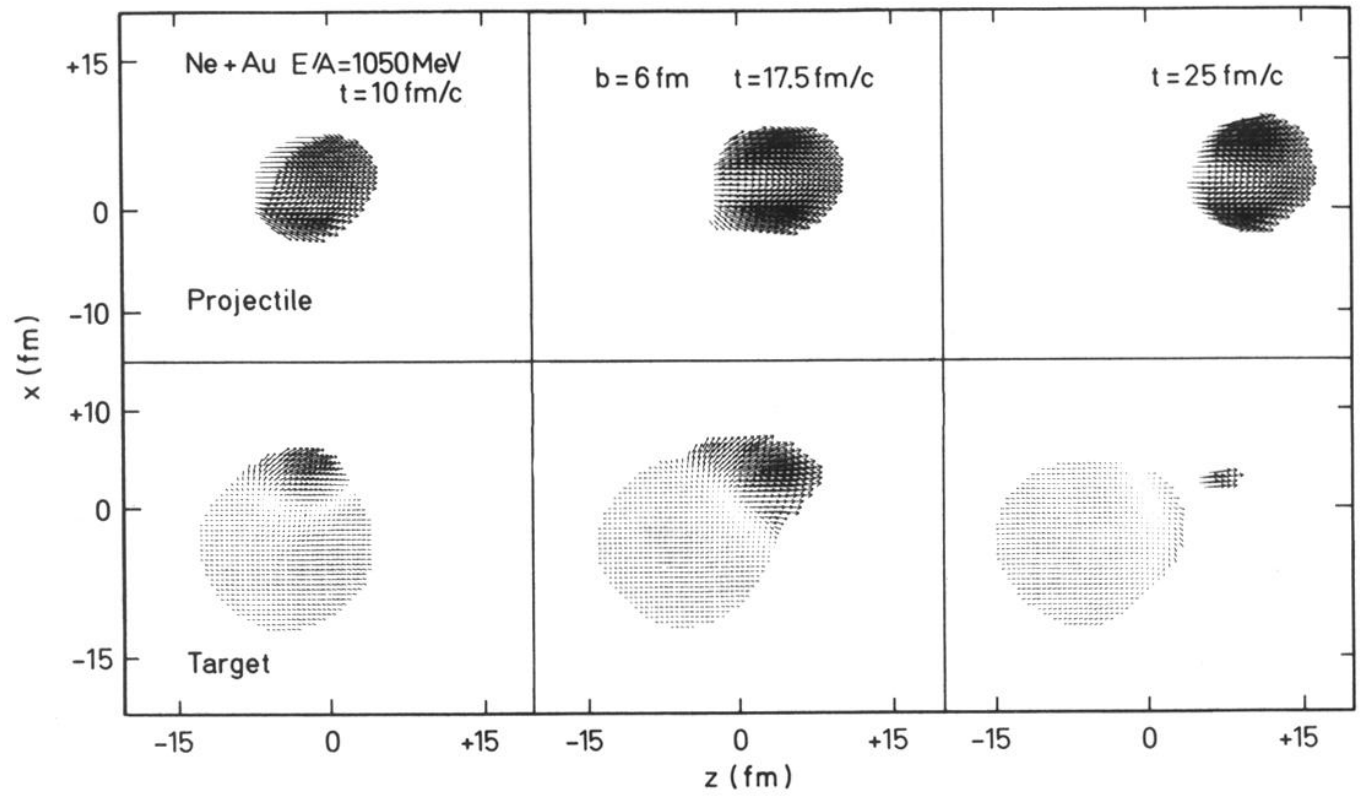

FIG. 19. Velocity profile for $b=6 \mathrm{fm}$ in the $z x$ plane at the beginning of the reaction, $t=10-20 \mathrm{fm} / c$, in steps of $5 \mathrm{fm} / c$, separately displayed for projectile and target nucleons. The length and direction of the arrows are proportional to the velocity in the $z x$ plane. 